Pacific

Journal of

Mathematics

GROUPS ACTING ON CANTOR SETS AND THE END STRUCTURE OF GRAPHS

BRIAN H. Bowditch

Volume $207 \quad$ No. 1

November 2002 


\title{
GROUPS ACTING ON CANTOR SETS AND THE END STRUCTURE OF GRAPHS
}

\author{
BRIAN H. BOWDITCH
}

\begin{abstract}
We describe a variation of the Bergman norm for the algebra of cuts of a connected graph admitting a cofinite group action. By a construction of Dunwoody, this enables us to obtain nested generating sets for invariant subalgebras. We describe a few applications, in particular, to convergence groups acting on Cantor sets. Under certain finiteness assumptions one can deduce that such actions are necessarily geometrically finite, and hence arise as the boundaries of relatively hyperbolic groups. Similar results have already been obtained by Gerasimov by other methods. One can also use these techniques to give an alternative approach to the Almost Stability Theorem of Dicks and Dunwoody.
\end{abstract}

\section{Introduction.}

In this paper, we describe some of the interconnections between the end structure of graphs, groups acting on protrees, and convergence actions on Cantor sets. Our work ties in with recent work of Gerasimov and Dunwoody and earlier work of Bergman.

It was shown by Hopf in the 1930s that the space of ends of (any Cayley graph of) a finitely generated group, $\Gamma$, either consists of 0,1 or 2 points, or else is a Cantor set. In the late 1960s, Stallings used topological methods to show that in the last case $\Gamma$ splits nontrivially over a finite subgroup. Shortly afterwards, Bergman [Ber] gave a different proof of the same result. One can interpret his construction as giving us a $\Gamma$-invariant nested subset of the Boolean algebra of clopen sets of the space of ends, and hence a splitting of the group via Bass-Serre theory. In fact, one can think of this algebra combinatorially in terms of cuts of the Cayley graph, i.e., finite sets of edges which separate the graph into more than one infinite subset (cf. [Du1]). Bergman's proof uses a certain "norm" defined on the set of such cuts. Recently Dunwoody and Swenson showed how one can use Bergman's norm to construct nested generating sets of arbitrary invariant subalgebras of the algebra of cuts of a locally finite graph (see [DuS] and Section 8). In this paper we generalise Bergman's norm, and some its consequences, to certain non-locally finite graphs (see Section 9). One of the main applications we 
describe here will be to convergence group actions on Cantor sets. Similar results have already been obtained by Gerasimov [Ger], by other methods. One can also use these ideas to give a simplified proof of the Almost Stability Theorem of [DiD] (see Section 14).

The notion of a convergence group was defined by Gehring and Martin [GehM]. It extracts the essential dynamical features of a kleinian group acting on the boundary of (classical) hyperbolic space. This generalises to boundaries of proper hyperbolic spaces in the sense of Gromov $[\mathbf{G r}]$, see for example, $[\mathbf{T} \mathbf{2}, \mathbf{F}, \mathbf{B o 3}]$. The celebrated result of $[\mathbf{T 1}, \mathbf{G a}, \mathbf{C J}]$ tells us that every convergence action on the circle arises from a fuchsian group. (In particular, the work of Tukia [T1] deals with the case of cyclically ordered Cantor sets.) There has also been a study of convergence actions on more general continua and their applications to hyperbolic and relatively hyperbolic groups (see for example [Bo2] and the references therein). Here we turn our attention to the opposite extreme, namely convergence actions on Cantor sets. An extensive study of such actions in relation to median algebras can be found in [Ger]. An example of such an action is that of a finitely generated group acting on its space of ends, which is a Cantor set if the group is not virtually cyclic and splits nontrivially over a finite subgroup. We shall see that, under some finiteness assumptions on the group, this type of example is typical.

Via Stone duality, a convergence action of a group, $\Gamma$, on a Cantor set is equivalent to a certain kind of action on a Boolean algebra. To obtain a splitting of $\Gamma$ from this action, there are two issues to be addressed. The first is to find a $\Gamma$-invariant nested generating set for the Boolean algebra, and the second is to arrange that this set is cofinite (in other words to show that the algebra is finitely generated as a $\Gamma$-Boolean algebra). To get a handle on these issues, we assume that $\Gamma$ is (relatively) finitely generated, and work with the algebra of cuts of a connected $\Gamma$-graph given by this hypothesis. The nested generating set is obtained using the Bergman norm (see Sections 8 and 9). In [Ger], a similar result is obtained via the theory of median algebras. For the second part, we need to assume that $\Gamma$ is (relatively) almost finitely presented. We use a result of $[\mathbf{D i D}]$ or of $[\mathbf{B e s F}]$ to obtain a cofinite generating set (Section 11). This is really an issue of accessibility. (We remark that it is shown in $[\mathbf{D i D}]$ that a finitely generated group, $\Gamma$, is accessible if and only if the Boolean algebra of almost invariant subsets is finitely generated as a $\Gamma$-Boolean algebra.)

I am indebted to Victor Gerasimov for explaining to me some of his work in this area. I have benefited greatly from discussions with Martin Dunwoody, in particular for introducing me to the work of Bergman, as well as explaining to me how accessibility results could be used to obtain finitely generated algebras (see Sections 10 and 11). Some of the work for the present article was carried out while visiting the Centre de Recerca Matemàtica in 
Barcelona. I am grateful for the support and hospitality of this institution, and for helpful discussions with Warren Dicks while there.

\section{Summary of results.}

We shall introduce some of the results of this paper by giving a series of results, which are, in some sense, increasingly general, but which require greater elaboration in their formulation. They will be proven is Section 12 . As mentioned in the introduction, most of these results have been obtained, in some form, by Gerasimov using median algebras [Ger]. The results will be made more precise in later sections. We shall assume throughout that the convergence actions are minimal, i.e., that there is no discontinuity domain. For definitions regarding convergence actions, see Section 4.

The simplest example of a convergence action on a Cantor set is that of a (virtually) free group acting on its space of ends (which is the same as its boundary as a hyperbolic group). Such an action has no parabolic points. One result says that this is typical:

Theorem 1.1. If $\Gamma$ is an almost finitely presented group acting as a minimal convergence group on a Cantor set, $M$, without parabolic points, then $\Gamma$ is virtually free, and there is a $\Gamma$-equivariant homeomorphism from $M$ to the space of ends of $\Gamma$.

By almost finitely presented we mean that $\Gamma$ admits a cocompact properly discontinuous action on a locally finite connected 2-complex, $\Sigma$, with $H_{1}\left(\Sigma ; \mathbb{Z}_{2}\right)=0$. (Without loss of generality, one can assume that the action on $\Sigma$ is also free.) Clearly, finite presentability in the usual sense implies almost finite presentability.

More generally, it is well-known that any almost finitely generated group acts as a convergence group on its space of ends (see Section 5). If $\Gamma$ is finitely presented, then Dunwoody's accessibility theorem [Du2] tells us that we can represent $\Gamma$ as a finite graph of groups with finite edge groups and finite or one-ended vertex groups. The one-ended vertex groups are uniquely determined (up to conjugacy) as the maximal one-ended subgroups of $\Gamma$, and are precisely the maximal parabolic subgroups with respect to this convergence action. We have a converse:

Theorem 1.2. Suppose that $\Gamma$ is an almost finitely presented group and acts as a minimal convergence group on a Cantor set, $M$, with all maximal parabolic subgroups finitely generated and one-ended. Then, $M$ is equivariantly homeomorphic to the space of ends of $\Gamma$.

The hypothesis on parabolic subgroups in the above result is somewhat unnatural. Note that the splitting given by Dunwoody's accessiblity result gives us, via Bass-Serre theory, an action on a simplicial tree with finite edge stabilisers and finite quotient. To such a tree, we can associate a 
"boundary" as described in Section 6. The group acts as a convergence group on this boundary, with the infinite vertex stabilisers as the maximal parabolic subgroups. If these happen to be one-ended, then we recover the space of ends of $\Gamma$. We also have a converse:

Theorem 1.3. Suppose that $\Gamma$ is an almost finitely presented group and acts as a minimal convergence group on a Cantor set, $M$. Then, $\Gamma$ has a representation as a finite graph of groups with finite edge groups such that $M$ is equivariantly homeomorphic to the "boundary" of the associated BassSerre tree.

In fact, we only really require that $\Gamma$ be almost finitely presented relative to a set of parabolic subgroups (see Section 2 for a definition). More precisely:

Theorem 1.4. Suppose that a group, $\Gamma$, acts as a minimal convergence group on a Cantor set, $M$. Suppose that $\mathcal{G}$ is a finite collection of parabolic subgroups of $\Gamma$ with respect to this action. Suppose that $\Gamma$ is almost finitely presented relative to $\mathcal{G}$. Then, the conclusion of Theorem 1.3 holds.

Note that, from the conclusion of Theorem 1.3, one may deduce that there are only finitely many conjugacy classes of maximal parabolic subgroups. In fact, it follows that the action of $\Gamma$ on $M$ is geometrically finite, and that $\Gamma$ is hyperbolic relative to the collection of maximal parabolic subgroups (see Section 4 for definitions). Moreover, the boundary of $\Gamma$ as a relatively hyperbolic group may be identified with the boundary of the Bass-Serre tree. Note that in Theorem 1.4, the splitting obtained is "relative to" $\mathcal{G}$, i.e., each element of $\mathcal{G}$ is conjugate into a vertex group.

We can further weaken the hypotheses, and assume only that $\Gamma$ is finitely generated (relative to a class of parabolic subgroups). However, in this case we can only be assured of an action of $\Gamma$ on a protree, as opposed to a simplicial tree. From this, one can deduce that the original action on $M$ is an inverse limit of geometrically finite actions of the above type. This is elaborated on in Section 13.

\section{Finiteness conditions.}

In this section, we elaborate on the finiteness conditions featured in Section 1 . These are most conveniently expressed in terms of group actions on sets (cf. [Bo5]).

Suppose $\Gamma$ is a group. A $\Gamma$-set, $V$, is a set on which the group $\Gamma$ acts. Given $x \in V$, we write $\Gamma(x)=\{g \in \Gamma \mid g x=x\}$. We write $V_{\infty}=\{x \in V \mid$ $|\Gamma(x)|=\infty\}$. We can interpret a property of $V$ as a property of the group, $\Gamma$ "relative to" the set of infinite point stabilisers, $\left\{\Gamma(x) \mid x \in V_{\infty}\right\}$. We shall also speak of " $\Gamma$-graphs", " $\Gamma$-trees" and " $\Gamma$-Boolean algebras" etc. for such objects admitting $\Gamma$-actions. 
We shall say that the $\Gamma$-set, $V$, is cofinite if $|V / \Gamma|$ is finite. A pair stabiliser is a subgroup of the form $\Gamma(x) \cap \Gamma(y)$ for $x \neq y$. We shall say that $V$ is "0-connected" if it can be identified with the vertex set, $V(K)$, of a connected cofinite $\Gamma$-graph, $K$ (or equivalently, as a $\Gamma$-invariant subset of $V(K)$ containing $\left.V_{\infty}(K)\right)$. We say that $V$ is 1-connected if it is the vertex set of a cofinite simply connected $\mathrm{CW}$-complex (or equivalently, a $\Gamma$-invariant subset containing $V_{\infty}(\Sigma)$ of the vertex set of a cofinite simplicial complex, $\Sigma)$. Note that 1-connectedness is equivalent to the assertion that for some (or equivalently any) cofinite connected $\Gamma$-graph with vertex set $V$, there is some $n \geq 2$ such that $\Omega_{n}(K)$ is simply connected. Here $\Omega_{n}(K)$ is the 2 -complex obtained by attaching a 2-cell along every circuit of length at most $n$ in $K$. More generally, we say that $V$ is $\mathbb{Z}_{2}$-homologically 1 -connected if $\Omega_{n}(K)$ is $\mathbb{Z}_{2}$-acyclic, i.e., $H_{1}\left(\Omega_{n}(K) ; \mathbb{Z}_{2}\right)=0$.

We say that a graph, $K$, is fine if, for each $n$, there are only finitely many circuits of length $n$ containing any given edge. Clearly, this implies that the complex $\Omega_{n}(K)$ described above is locally finite away from $V=V(K)$ (and can thus be subdivided to give a simplicial complex that is locally finite away from $V$ ). We say that a $\Gamma$-set is fine if some (hence any) cofinite connected $\Gamma$-graph, $K$, with vertex set $V$, is fine. Here, the fineness of $K$ is equivalent to saying that there are finitely many circuits of any given length modulo $\Gamma$.

Note that if $V$ is fine and ( $\mathbb{Z}_{2}$-homologically) 1-connected we can embed $V$ equivariantly in the vertex set, $V(\Sigma)$, of a cocompact simply connected $\left(\mathbb{Z}_{2}\right.$-acyclic) 2-dimensional simplicial complex, $\Sigma$, which is locally finite away from $V(\Sigma)$, and such that the stabiliser of each element of $V(\Sigma) \backslash V$ is finite.

Suppose that $\mathcal{G}$ is a nonempty collection of self-normalising subgroups of $\Gamma$, which is a finite union of conjugacy classes, and such that the intersection of any two distinct elements of $\mathcal{G}$ is finite. We may view $\mathcal{G}$ as a $\Gamma$-set with $\Gamma$ acting by conjugation. We say that $\Gamma$ is finitely generated relative to $\mathcal{G}$ if $\mathcal{G}$ is 0 -connected. We say that $\Gamma$ is (almost) finitely presented relative to $\mathcal{G}$ if $\mathcal{G}$ is $\left(\mathbb{Z}_{2}\right.$-homologically) 1 -connected. In the case of interest to us, $\mathcal{G}$ will always be fine.

This ties in with the usual group theoretical terminology. A group, $\Gamma$, is finitely generated if and only if it admits a cofinite action on a locally finite simplicial 1-complex. It is (almost) finitely presented if and only if it admits a cofinite action on a ( $\mathbb{Z}_{2}$-homologically) 1-connected locally finite simplicial 2-complex. Here the actions can in fact be taken to be free.

If $G \subseteq \Gamma$ is self-normalising with presentation $\langle A ; R\rangle$, then $\Gamma$ is finitely generated relative to $G$ if and only if there is a finite set $B \subseteq \Gamma$, such that $\langle A \cup B\rangle$. It is finitely presented relative to $G$, if and only if there is a finite set of words, $S$, such that $\langle A \cup B ; R \cup S\rangle$ is a presentation of $\Gamma$. One can generalise this to a finite collection of self-normalising subgroups using the language of groupoids, though we shall not make that explicit here. 


\section{Boolean algebras.}

Let $\mathcal{B}$ be a Boolean ring, i.e., a commutative ring with a unity element, 1 , satisfying $x^{2}=x$ for all $x \in \mathcal{B}$. We write $x^{*}=1+x, x \wedge y=x y$ and $x \vee y=x+y+x y$. Thus, $(\mathcal{B}, \wedge, \vee, *)$ is a Boolean algebra. We write $x \leq y$ to mean that $x y=x$. Thus $\leq$ is a partial order on $\mathcal{B}$, and $\left[x \mapsto x^{*}\right]$ is an order reversing involution. Given any set, $X$, its power set, $\mathcal{P}(X)$ is a Boolean algebra with $P^{*}=X \backslash P, P \wedge Q=P \cap Q$ and $P \vee Q=P \cup Q$, for $P, Q \in \mathcal{P}(X)$. Suppose $M$ is a compact totally disconnected topological space. The set, $\mathcal{B}(M)$ of clopen subsets of $M$ is a Boolean subalgebra of $\mathcal{P}(M)$.

The Stone duality theorem [St] tells us that every Boolean algebra arises in this way (see for example $[\mathbf{S i}]$ ). Suppose that $\mathcal{B}$ is a Boolean algebra. We associate to $\mathcal{B}$ a compact totally disconnected space $\Xi=\Xi(\mathcal{B})$, called the Stone dual, such that $\mathcal{B}(\Xi) \cong \mathcal{B}$. This can be described in a number of equivalent ways. For example we can define $\Xi$ as the set of Boolean ring homomorphisms from $\mathcal{B}$ to $\mathbb{Z}_{2}$. This is a closed subset of the Tychonoff cube $\mathbb{Z}_{2}^{\mathcal{B}}$, and we topologise $\Xi$ accordingly.

Alternatively, we define $\Xi$ as the maximal ideal spectrum of the ring $\mathcal{B}$ with the Zariski topology. Note that the complement of a maximal ideal in $\mathcal{B}$ is an ultrafilter. We therefore get the same thing by taking the set of ultrafilters on $\mathcal{B}$. From this point of view, we can define a basis for the closed sets by taking a typical basis element to be the set of all ultrafilters that contain a given element of $\mathcal{B}$.

Suppose that $\mathcal{B}$ is a subalgebra of $\mathcal{P}(X)$ for some set $X$. If $x \in X$, then the ultrafilter $\mathcal{O}(x)=\{P \in \mathcal{B} \mid x \in P\}$ determines a point of $\Xi(\mathcal{B})$, so we get natural map from $X$ to $\Xi(\mathcal{B})$. If $M$ is a compact and totally disconnected topological space, and $\mathcal{B}=\mathcal{B}(M)$, then this map gives us the Stone isomorphism from $M$ to $\Xi(\mathcal{B}(M))$.

Note that if $f: \mathcal{B} \longrightarrow \mathcal{B}^{\prime}$ is a homomorphism, we get a continuous dual map, $f_{*}: \Xi\left(\mathcal{B}^{\prime}\right) \longrightarrow \Xi(\mathcal{B})$. If $f$ is surjective, then $f_{*}$ is injective, so we can identify $\Xi\left(\mathcal{B}^{\prime}\right)$ as a closed subset of $\Xi(\mathcal{B})$.

We say that two nonzero elements $x, y \in \mathcal{B}$ are nested if $x y$ is equal to 0 , $x, y$ or $1+x+y$. This is equivalent to saying that one of $x y, x y^{*}, x^{*} y$ or $x^{*} y^{*}$ equals 0 . We say that a subset, $\mathcal{E} \subseteq \mathcal{B}$ is nested if $0,1 \notin \mathcal{E}$ and every pair of elements of $\mathcal{E}$ are nested. Note that if $\mathcal{E}$ is nested, then so is the *-invariant set $\mathcal{E} \cup \mathcal{E}^{*}$.

\section{Convergence groups.}

The notion of a convergence group was defined in $[$ GehM $]$. For further discussion, see [T2, Bo3, T3].

Suppose that $M$ is a compact metrisable space and that $\Gamma$ is a group acting by homeomorphism on $M$. We say that this is a convergence action 
(or that $\Gamma$ is a convergence group) if the induced action on the space of distinct triples of $M$ (i.e., $M \times M \times M$ minus the large diagonal) is properly discontinuous. This is equivalent to the statement that if $\left(g_{n}\right)_{n \in \mathbb{N}}$ is any infinite sequence of distinct elements of $\Gamma$, then there are points, $a, b \in M$, and a subsequence $\left(g_{n_{i}}\right)_{i}$ such that $g_{n_{i}} \mid M \backslash\{a\}$ converges locally uniformly to $b$. We refer to the latter statement as the "convergence property" of $\Gamma$.

A subgroup, $G$, of $\Gamma$ is parabolic if it is infinite and fixes a unique point. Such a fixed point, $x$, is a called a parabolic point, and its stabiliser, $\Gamma(x)$, is a maximal parabolic subgroup of $\Gamma$. We say that $x$ is a bounded parabolic point if $(M \backslash\{x\}) / \Gamma(x)$ is compact. (We allow for the possibility of a parabolic group being an infinite torsion group.)

A point $x \in M$ is a conical limit point if there is a sequence of elements $g_{n} \in \Gamma$, and distinct points, $a, b \in M$ such that $g_{n} x \rightarrow a$ and $g_{n} y \rightarrow b$ for all $y \in M \backslash\{x\}$. It is shown in [T3] that a conical limit point cannot be a parabolic point. We say that the action of $\Gamma$ on $M$ is geometrically finite if every point of $M$ is either a conical limit point or a bounded parabolic point. Such actions have been studied by Tukia [T3].

By the space of distinct pairs of $M$, we mean $M \times M$ minus the diagonal.

Lemma 4.1. Suppose that $\Gamma$ acts on $M$ as a convergence group, and that $x, y \in M$ are distinct and not conical limit points. Then, $\Gamma(x) \cap \Gamma(y)$ is finite, and the $\Gamma$-orbit of $(x, y)$ is a discrete subset of the space of distinct pairs.

Proof. Suppose, for contradiction, that $g_{n} \in \Gamma$ is a sequence of distinct elements of $\Gamma$ with $g_{n} x \rightarrow a$ and $g_{n} y \rightarrow b$ with $a, b \in M$ distinct. The convergence property tells us that after passing to a subsequence, either $g_{n} \mid M \backslash\{x\}$ converges (locally uniformly) to $b$, or else $g_{n} \mid M \backslash\{y\}$ converges (locally uniformly) to $a$. Thus, either $x$ or $y$ is a conical limit point.

Recall the terms "connected" and "fine" as defined in Section 2.

Lemma 4.2. Suppose that $\Gamma$ acts on $M$ as a convergence group, and that $\Pi \subseteq M$ is a $\Gamma$-invariant subset. Suppose that no point of $\Pi$ is a conical limit point. If $\Pi$ is connected (as a $\Gamma$-set) then it is fine.

Proof. We show that if $K$ is any cofinite $\Gamma$-graph with vertex set $V(K)=\Pi$, then modulo $\Gamma$, there are only finitely many circuits of length $n$ for any given $n$. Since $\Pi$ is connected, we can take $K$ to be connected, and we see that $K$ is fine. Together with the first part of Lemma 4.1, this implies that $\Pi$ is fine as claimed.

Suppose, for contradiction, that $\left(\beta^{k}\right)_{k \in \mathbb{N}}$ is an infinite sequence of circuits of length $n$ in $K$, each lying in a different $\Gamma$-orbit. We write $\beta^{k}=x_{1}^{k} \ldots x_{n}^{k}$, taking subscripts $\bmod n$. Passing to a subsequence, we can suppose that for all $i$ each of the edges $\left\{x_{i}^{k} x_{i+1}^{k}\right\}_{k}$ lie in the same $\Gamma$-orbit. Thus, modulo $\Gamma$, we can suppose that $x_{0}^{k}=x_{0}$ and $x_{1}^{k}=x_{1}$ are independent of $k$. Now 
by Lemma 4.1 , the set of pairs $\left\{\left(x_{1}, x_{2}^{k}\right) \mid k \in \mathbb{N}\right\}$ is discrete in the space of distinct pairs. Thus, again after passing to a subsequence, we can suppose that either $x_{2}^{k}=x_{2}$ is constant, or that $x_{2}^{k} \rightarrow x_{1}$. In the latter case, we can suppose that the $x_{2}^{k}$ are all distinct, so since the set of pairs $\left(x_{2}^{k}, x_{3}^{k}\right)_{k}$ is discrete, we must also have $x_{3}^{k} \rightarrow x_{1}$. It follows inductively that $x_{i}^{k} \rightarrow x_{1}$ for all $i$, giving the contradiction that $x_{0}=x_{n}^{k} \rightarrow x_{1}$. We can thus assume that $x_{2}^{k}=x_{2}$ is constant. But now, the same argument tells us that $x_{3}^{k}$ is constant, so by induction, $x_{i}^{k}$ is constant for all $i$. We derive the contradiction that $\beta^{k}$ is constant.

Note that, by the result of Tukia [T3], Lemma 4.2 applies to a set of parabolic points.

A standard example of a convergence group is the action induced on the boundary, $\partial X$, by any properly discontinuous action of a group, $\Gamma$, on a proper (complete locally compact) hyperbolic space, $X$. If the action on $\partial X$ is geometrically finite, we say that $\Gamma$ is hyperbolic relative to the set, $\mathcal{G}$, of maximal parabolic subgroups of $\Gamma$. (In [Bo5], we impose the additional requirement that each element of $\mathcal{G}$ be finitely generated, but this need not concern us here.) It is necessarily the case that $\mathcal{G}$ is cofinite and connected (and hence fine) as a $\Gamma$-set. Relatively hyperbolic groups were introduced by Gromov $[\mathbf{G r}]$. It turns out that they can be characterised dynamically as geometrically finite convergence groups $[\mathbf{Y}]$.

We note that, in the case where $M$ is totally disconnected, we can express the convergence property in terms of the Boolean algebra, $\mathcal{B}=\mathcal{B}(M)$ of clopen subsets of $M$. By a ternary partition of $\mathcal{B}$, we mean a triple of pairwise disjoint nonzero elements, $A, B, C \in \mathcal{B}$ such that $A+B+C=1$. (In other words, $M=A \sqcup B \sqcup C$.) Note that $A \times B \times C$ is a compact subset of the space of distinct triples of $M$.

Suppose that $\Gamma$ acts by isomorphism on a Boolean algebra $\mathcal{B}$.

Definition. We say that the action is a convergence action if, for any two ternary partitions, $\left(a_{1}, a_{2}, a_{3}\right)$ and $\left(b_{1}, b_{2}, b_{3}\right)$ of $\mathcal{B}$, the set $\left\{g \in \Gamma \mid b_{1} \wedge g a_{1} \neq\right.$ $\left.0, b_{2} \wedge g a_{2} \neq 0, b_{3} \wedge g a_{3} \neq 0\right\}$ is finite.

To see that this agrees with the notion already defined for $M$, note that compact subset of the set of distinct triples of $M$ can be finitely covered by sets of the form $A \times B \times C$, where $A, B, C$ is a ternary partition of $M$.

We finally note that if $M$ is an inverse limit of compact spaces, $\left(M_{n}\right)_{n \in \mathbb{N}}$, each admitting a $\Gamma$-action that commutes with the inverse limit system, then the action of $\Gamma$ on $M$ is a convergence action if and only if the action on each $M_{n}$ is a convergence action. 


\section{Ends of graphs.}

In this section, we explain how to associate a "space of ends", $\Xi_{0}(K)$, to a connected graph, $K$.

Let $K$ be a connected graph, with vertex set $V=V(K)$, and edge set $E(K)$. Let $V_{0}=V_{0}(K)$ be the set of vertices of finite degree. If $W \subseteq V(K)$ we write $I(W)$ for the set of edges with precisely one endpoint in $W$.

Definition. A subset $W \subseteq V(K)$ is a $K$-break if $I(W)$ is finite.

We write $\mathcal{B}=\mathcal{B}(K)$ for the Boolean algebra of $K$-breaks. We write $\Xi(K)=\Xi(\mathcal{B})$ for the Stone dual of $\mathcal{B}$.

There is a natural map, $\xi: V \longrightarrow \Xi(K)$, defined by sending $x \in V$ to the ultrafilter of elements of $\mathcal{B}$ containing $x$. Note that $\xi \mid V_{0}$ is injective, and every point of $\xi\left(V_{0}\right)$ is isolated in $\Xi(K)$. We write $\Xi_{0}(K)=\Xi(K) \backslash \xi\left(V_{0}\right)$. This is a closed subset of $\Xi(K)$. Note that if $K$ is locally finite, then $\Xi_{0}(K)$ is the precisely the space of ends of $K$ in the usual sense.

Another way to define $\Xi_{0}(K)$ is as follows. Let $\mathcal{I}$ be the ideal of $\mathcal{B}$ consisting of finite subsets of $V$, and let $\mathcal{C}(K)$ be the quotient $\mathcal{B} / \mathcal{I}$. There is an inclusion of $\Xi(\mathcal{C})$ in $\Xi(\mathcal{B})$ whose image is precisely $\Xi_{0}(K)$.

We shall say that $K$ is one-ended if $\Xi_{0}(K)$ consists of a single point, i.e., no finite set of edges separates $K$ into two or more infinite subgraphs.

Lemma 5.1. If a group $\Gamma$ acts on a connected graph, $K$, with finite edge stabilisers, then the induced action of $\Gamma$ on $\Xi(K)$ is a convergence action.

Proof. Note that if $F \subseteq E(K)$ is any finite set of edges, then $\{g \in \Gamma \mid$ $F \cap g F \neq \emptyset\}$ is finite.

Suppose that $\left(A_{1}, A_{2}, A_{3}\right)$ and $\left(B_{1}, B_{2}, B_{3}\right)$ are ternary partitions of $\mathcal{B}$. Let $I=I\left(A_{1}\right) \cup I\left(A_{2}\right) \cup I\left(A_{3}\right)$ and $J=I\left(B_{1}\right) \cup I\left(B_{2}\right) \cup I\left(B_{3}\right)$. Let $X$ and $Y$ be any connected finite subgraphs of $K$ containing $I$ and $J$ respectively.

Suppose that $E(X) \cap E(Y)=\emptyset$. Now, $I \cap E(Y)=\emptyset$ and so all the vertices of $Y$ must lie in the same element of $\left\{A_{1}, A_{2}, A_{3}\right\}$, say $V(Y) \subseteq A_{i}$. Similarly, $V(X) \subseteq B_{j}$ for some $j$. We claim that $V(K) \subseteq A_{i} \cup B_{j}$. For suppose $x \in V(K) \backslash\left(A_{i} \cup B_{j}\right)$. Let $\alpha$ be a shortest path connecting $x$ to $A_{i} \cup B_{j}$. Let $y, z$ be, respectively, the last and last but one vertices of $\alpha$. Without loss of generality, $y \in A_{i}$. Now $z \notin A_{i}$, and so the edge connecting $y$ and $z$ must lie in $I \subseteq E(X)$. It follows that $z \in V(X) \subseteq B_{j}$, giving a contradiction, and hence proving the claim. Note that if $k \neq i, j$, then $A_{k} \cap A_{i}=B_{k} \cap B_{j}=\emptyset$, so that $A_{k} \cap B_{k}=\emptyset$.

Now if $g \in \Gamma$ with $B_{i} \cap g A_{i} \neq \emptyset$ for each $i=1,2,3$, then, by the previous paragraph, we see that $E(Y) \cap g E(X) \neq \emptyset$. But the set of such $g$ for given $\left(A_{1}, A_{2}, A_{3}\right)$ and $\left(B_{1}, B_{2}, B_{3}\right)$ is finite. This shows that $\Gamma$ is a convergence group as claimed. 
In particular, we deduce the well-known fact that any finitely generated group acts a convergence group on its space of ends. (Take $K$ to be any Cayley graph of $\Gamma$.)

Suppose that $f: K \longrightarrow L$ is a contraction onto a connected graph, $L$ (i.e., a map such that the preimage of each edge of $L$ is an edge of $K$, and the preimage of every vertex of $L$ is a connected subgraph of $K)$. There is a natural inclusion of $\mathcal{B}(L)$ into $\mathcal{B}(K)$. If the preimage of every finitedegree vertex of $L$ is finite, then this descends to an injection from $\mathcal{C}(L)$ to $\mathcal{C}(K)$. If, in addition, the preimage of every infinite degree vertex of $L$ is one-ended, then this is an isomorphism, so that $\Xi_{0}(K)$ and $\Xi_{0}(L)$ are canonically homeomorphic.

\section{Simplicial trees.}

Let $T$ be a simplicial tree with vertex set $V=V(T)$, edge set $E(T)$, and directed edge set $\vec{E}(T)$. Given $\vec{e} \in \vec{E}(T)$, we write $e$ for the underlying undirected edge, and $-\vec{e}$ for the same edge pointing in the opposite direction. Let $V(\vec{e}) \subseteq V$ be the set of vertices, $v$, such that $\vec{e}$ points towards $v$. If $\vec{e}, \vec{f} \in \vec{E}(T)$, we write $\vec{e}<\vec{f}$ to mean that $\vec{f}$ points towards $\vec{e}$ and $\vec{e}$ points away from $\vec{f}$. (In some papers the opposite convention is used.) Note that this is equivalent to saying that $V(\vec{e})$ is strictly contained in $V(\vec{f})$. Clearly $\leq$ is a partial order on $\vec{E}(T)$ with order reversing involution $[\vec{e} \mapsto-\vec{e}]$.

A subset, $F \subseteq \vec{E}(T)$ is a transversal if, for all $\vec{e} \in \vec{E}(T)$, precisely one of $\vec{e}$ or $-\vec{e}$ lies in $F$. A transversal, $F$, is a flow on $T$ if no two elements of $F$ point away from each other (i.e., there do not exist $\vec{e}, \vec{f} \in F$ with $\vec{e} \leq-\vec{f}$ ). A flow must be of one of two types. Either there is some (unique) $v \in V(T)$ such that each element of $F$ points towards $v$, or else there is some infinite ray, $\alpha \subseteq T$ such that all edges of $F \cap E(\alpha)$ point away from its basepoint, and all other elements of $F$ point towards $\alpha$. We can identify the set of flows of the second kind with the boundary, $\partial T$, of $T$, thought of as a hyperbolic space in the sense of Gromov $[\mathbf{G r}]$.

Recall the definitions of $\mathcal{B}=\mathcal{B}(T)$ and $\Xi(T)$ from Section 5. Note that $\mathcal{E}=\mathcal{E}(T)=\{V(\vec{e}) \mid \vec{e} \in \vec{E}(T)\}$ is a nested set of generators for $\mathcal{B}$. (The partial order, $\leq$, on $\vec{E}(T)$ agrees with that on $\mathcal{E} \subseteq \mathcal{B}$, and the involution $[\vec{e} \mapsto-\vec{e}]$ corresponds to the involution $\left[W \mapsto W^{*}\right]$.) We can think of an element of $\Xi(T)$ as an ultrafilter on $\mathcal{B}(T)$. The elements of $\mathcal{E}$ lying in that ultrafilter define a flow on $T$. In this way we may identify $\Xi(T)$ with the set of flows on $T$, and hence with $V(T) \sqcup \partial T$.

Now, $T$ is fine (as defined in Section 2) and hyperbolic (in the sense of Gromov). It thus has associated with it a compact space $\Delta T$, as in [Bo5]. As a set, $\Delta T$ may be defined as $V(T) \sqcup \partial T$. Note that any two elements, $x, y \in \Delta T$ can be connected by a unique arc, $[x, y]$, which may be compact, 
a ray, or a bi-infinite geodesic depending on whether or not $x, y \in V(T)$. Given $x \in \Delta T$ and $I \subseteq E(T)$, let $B(x, I)=\{y \in \Delta T \mid I \cap E([x, y])=\emptyset\}$. We define a topology on $\Delta T$ by taking a neighbourhood base of $x \in \Delta(T)$ to be the collection $\{B(x, I)\}_{I}$ as $I$ runs over all finite subsets $E(T)$. In this topology, $\Delta T$ is compact and Hausdorff. We see that, as sets, $\Delta T$ can be identified with $\Xi(T)$, and one can readily verify that the two topologies agree.

As with more general graphs (Section 5), the isolated points of $\Delta T \equiv \Xi(T)$ are precisely the vertices of $T$ of finite degree. We shall define the ideal boundary $\Delta_{0} T$, of the tree, $T$, as the space $\Delta T$ minus the vertices of $T$ of finite degree. Again, this can be identified with with $\Xi_{0}(T)$ as defined in Section 5.

If a group $\Gamma$ acts on a simplicial tree, $T$, with finite edge stabilisers, then the induced action on $\Xi(T) \equiv \Delta T$ is a convergence action. In fact, if the action of $\Gamma$ on $T$ is cofinite, then the action on $\Delta T$ is geometrically finite, with the infinite vertex groups precisely the maximal parabolic subgroups. Thus, $\Gamma$ is hyperbolic relative to the infinite vertex groups, and its boundary can be identified with $\Xi_{0}(T) \equiv \Delta_{0} T$ (see [Bo5]). Moreover, if $\Gamma$ is finitely generated, and each infinite vertex group is finitely generated and one-ended, then from the last paragraph of Section 5 , we see that $\Delta_{0} T$ is canonically homeomorphic to the space of ends of $\Gamma$.

\section{Protrees and nested sets.}

A protree is a set $\Theta$, with an involution $\left[x \mapsto x^{*}\right]$ and a partial order, $\leq$, with the property that for any $x, y \in \Theta$, precisely one of the six relations $x<y, x^{*}<y, x<y^{*}, x^{*}<y^{*}, x=y, x=y^{*}$ holds. This notion is due to Dunwoody (see for example [Du4]). We refer to a $*$-invariant subset of $\Theta$ as a subprotree.

An example of a protree is the directed edge set of any simplicial tree, as described in the last section. In fact, any finite protree can be realised as the directed edge set of a finite tree (a property which could serve as an equivalent definition). More generally any "discrete" protree can be realised as the directed edge set of a simplicial tree. A protree is said to be discrete if, for all $x, y \in \Theta$, the set $\{z \in \Theta \mid x \leq z \leq y\}$ is finite. We can define transversals and flows on a protree, exactly as for simplicial trees. (However, we cannot in general classify flows in the same way.)

Suppose $\Theta$ is a protree. Let $\mathcal{F}=\mathcal{F}(\Theta)$ be the Boolean ring with generating set $\Theta$, and with relations $x+x^{*}=1$ for all $x \in \Theta$ and $x y=0$ for all $x, y \in \Theta$ satisfying $x<y^{*}$ in $\Theta$. (It follows that $x<y^{*}$ also in $\mathcal{F}(\Theta)$ in the sense defined in Section 3.) Note that it follows that if $x, y \in \Theta$ are distinct, then $x y$ must be equal to $0, x, y$ or $1+x+y$. In particular, it follows that any element of $\mathcal{F}$ can be written as a sum of finitely many elements of $\Theta$. 
Note that if $x \in \Theta$, then we can define an epimorphism, $\theta: \mathcal{F} \longrightarrow \mathbb{Z}_{2}$ by $\theta(x)=1$ and $\theta(y)=0$ for all $y \in \Theta \backslash\{x\}$. It therefore follows that $x \neq 0$ for all $x \in \Theta$.

Suppose that $F \subseteq \Theta$ is any transversal. We may identify $F$ as a subset of $\mathcal{F}(\Theta)$, and as such, it generates $\mathcal{F}(\Theta)$ as a ring with unity (indeed as an additive group if we include also 1 ). If $x, y \in F$, then precisely one of the relations $x y=0, x^{*} y=0, x y^{*}=0, x^{*} y^{*}=0$ holds. Thus, $\Theta$ is a nested set of generators for $\mathcal{F}(\Theta)$. Any element of $\mathcal{F}(\Theta)$ can be written in a standard form $\epsilon+\sum_{i=1}^{n} x_{i}$ where $\epsilon \in\{0,1\}$ and $x_{1}, \ldots, x_{n}$ are distinct elements of $F$.

If $\Theta$ is a discrete protree, and $T$ is the corresponding simplicial tree, then there is an epimorphism $\phi: \mathcal{F}(\Theta) \longrightarrow \mathcal{B}(T)$ defined by $\phi(x)=V(\vec{e})$, where $\vec{e} \in \vec{E}(T)$ is the directed edge corresponding to $x \in \Theta$. Let $F \subseteq \Theta$ be any transversal. If $x_{1}, \ldots, x_{n} \in F$ are distinct, then the corresponding elements of $\mathcal{B}(T)$ are distinct. If $n \neq 0$, it follows easily that their symmetric difference can be neither $\emptyset$ nor $V(T)$. In other words, we see that any standard form of any element in the kernel of $\phi$ must be trivial. Hence, the kernel is trivial, so $\phi$ is, in fact, an isomorphism.

We now return to the case of a general protree, $\Theta$, with transversal $F$. Suppose that $\Phi \subseteq \Theta$ is any subprotree. Then, there is a natural epimorphism, $\theta: \mathcal{F}(\Theta) \longrightarrow \mathcal{F}(\Phi)$ defined by $\theta(x)=x$ if $x \in \Phi$, and $\theta(x)=0$ if $x \in \Theta \backslash \Phi$. In particular, suppose $x_{1}, \ldots, x_{n}$ are distinct. We get an epimorphism from $\mathcal{F}(\Theta)$ to $\mathcal{F}(\Phi)$, where $\Phi=\bigcup_{i=1}^{n}\left\{x_{i}, x_{i}^{*}\right\}$. Now, $\mathcal{F}(\Phi)$ is isomorphic to the Boolean algebra on a finite tree, as above, and so it follows that $x_{1}+\cdots+x_{n} \notin\{0,1\}$. We see that the standard form of an element of $\Theta$ (with respect to a given transversal, $F$ ) is unique. We therefore have an explicit description of the ring $\mathcal{F}(\Theta)$.

If $\Phi \subseteq \Theta$ is again any subprotree, we also get a homomorphism from $\mathcal{F}(\Phi)$ to $\mathcal{F}(\Theta)$ which extends the inclusion of $\Phi$ in $\Theta$. From the above description, it is clear that this is injective. We therefore get a surjective map from $\Xi(\Theta)$ to $\Xi(\Phi)$. If $\Theta$ is an increasing union of subprotrees, $\left(\Theta_{n}\right)_{n \in \mathbb{N}}$, then it is easily seen that $\Xi(\Theta)$ in an inverse limit of the system $\left(\Xi\left(\Theta_{n}\right)\right)_{n}$ of topological spaces.

Let $\Xi(\Theta)=\Xi(\mathcal{F}(\Theta))$ be the Stone dual. If we think of an element of $\Xi(\Theta)$ as an ultrafilter on $\mathcal{F}(\Theta)$, then its intersection with $\Theta$ is a flow on $\Theta$. We may therefore identify $\Xi(\Theta)$ with the set of flows on $\Theta$. For non-discrete protrees, however, we do not get a clear distinction between vertices and boundary points, as in the simplicial case.

Suppose that $\mathcal{B}$ is any Boolean algebra with a nested set of generators, $\mathcal{E} \subseteq \mathcal{B}$. Now, $\mathcal{E}$ has the structure of a protree, with involution and partial order induced from $\mathcal{B}$. We can therefore construct the Boolean algebra $\mathcal{F}=\mathcal{F}(\mathcal{E})$ as above, and identify $\mathcal{B}$ as a quotient of $\mathcal{F}$. Note that $\Xi(\mathcal{B})$ can thus be identified as a closed subset of the space $\Xi(\mathcal{E})$. 
In fact, we can say more than this. We can formally identify $\mathcal{E}$ as a subset of $\mathcal{F}$. When composed with the canonical epimorphism from $\mathcal{F}$ to $\mathcal{B}$, this gives the inclusion of $\mathcal{E}$ into $\mathcal{B}$. Let $\mathcal{I}$ be the kernel of the canonical epimorphism from $\mathcal{F}$ to $\mathcal{B}$. Note that if $x, y \in \mathcal{E}$, then $x, y, x+y \notin \mathcal{I}$ (since, by the definition of a nested set, they correspond to distinct nonzero elements of $\mathcal{B}$ ). Now a combinatorial argument shows that $\mathcal{I}$ is generated by elements of the form $1+\sum_{i=1}^{n} x_{i}$, where $x_{1}, \ldots, x_{n} \in \mathcal{E}$ have the property that $x_{i} x_{j}=0$ for $i \neq j$, and if $y \in \mathcal{E}$ then for some $i \in\{1, \ldots, n\}$, we have $y x_{i} \in\left\{x_{i}, 1+x_{i}+y\right\}$. In other words, we can think of the elements $x_{1}, \ldots, x_{n}$ as a set of edges whose tails all meet at a "vertex" of degree $n$ of the protree $\mathcal{E}$. (This statement is precise if the protree $\mathcal{E}$ happens to be discrete, and hence the edge set of a simplicial tree.) Suppose that $a \in \Xi(\mathcal{E}) \backslash \Xi(\mathcal{B})$. Now $a$ corresponds to a flow, $F$, on $\mathcal{E}$. This cannot be identically zero on $\mathcal{I}$ and so must be nonzero on some generator of $\mathcal{I}$ of the above form. From this, it is easy to see that $F$ converges to some vertex of finite degree. But such a point is easily seen to be isolated in $\Xi(\mathcal{E})$. We have shown that every point of $\Xi(\mathcal{E}) \backslash \Xi(\mathcal{B})$ is isolated.

Finally, suppose that $\mathcal{B}$ is a subalgebra of $\mathcal{P}(V)$ for some set, $V$. There is a natural map from $V$ to $\Xi(\mathcal{B})$ as defined in Section 3. We therefore get a map from $V$ to $\Xi(\mathcal{E})$. The image of a point $x \in V$ in $\Xi(\mathcal{E})$ is defined by the flow $\{A \in \mathcal{E} \mid x \in A\}$ on $\mathcal{E}$.

\section{Construction of nested generating sets.}

It was shown in $[\mathbf{D u S}]$ how the Bergman norm can be used to construct invariant nested subsets of a Boolean algebra. Dunwoody has observed how an elaboration of this argument in fact gives us nested generating sets. The central idea may be formulated in a general fashion as follows.

Let $\mathcal{B}$ be a Boolean ring. We say that two elements, $x, y \in \mathcal{B}$ are disjoint if $x y=0$.

Suppose that $S$ is an ordered abelian group (or cancellative semigroup). Suppose that to each disjoint pair, $x, y \in \mathcal{B}$, we have associated an element $\sigma(x, y) \in S$. We suppose that $\sigma(x, y)=\sigma(y, x) \geq 0$, and that $\sigma(x, y)>0$ if $x, y \neq 0$. Moreover, if $x, y, z \in \mathcal{B}$ are pairwise disjoint, then $\sigma(x, y+$ $z)=\sigma(x, y)+\sigma(x, z)$. Given any $x, y \in \mathcal{B}$, we write $\mu(x)=\sigma\left(x, x^{*}\right)$ and $\mu(x \mid y)=\sigma\left(x y, x^{*} y\right)$. Clearly $\mu\left(x^{*}\right)=\mu(x)$ and $\mu\left(x^{*} \mid y\right)=\mu(x \mid y)$.

Suppose now that $x, y \in \mathcal{B}$ are non-nested, i.e., that $x y, x^{*} y, x y^{*}, x^{*} y^{*}$ are all nonzero.

If $\mu(y \mid x) \leq \mu\left(x \mid y^{*}\right)$, then

$$
\begin{aligned}
\mu(x y) & =\sigma\left(x y,(x y)^{*}\right) \\
& =\sigma\left(x y, x^{*}+x y^{*}\right) \\
& =\sigma\left(x y, x^{*}\right)+\sigma\left(x y, x y^{*}\right)
\end{aligned}
$$




$$
\begin{aligned}
& \leq \sigma\left(x y, x^{*}\right)+\sigma\left(x y^{*}, x^{*} y^{*}\right) \\
& <\sigma\left(x y, x^{*}\right)+\sigma\left(x y^{*}, x^{*} y^{*}\right)+\sigma\left(x y^{*}, x^{*} y\right) \\
& =\sigma\left(x y, x^{*}\right)+\sigma\left(x y^{*}, x^{*} y^{*}+x^{*} y\right) \\
& =\sigma\left(x y, x^{*}\right)+\sigma\left(x y^{*}, x^{*}\right) \\
& =\sigma\left(x, x^{*}\right) \\
& =\mu(x) .
\end{aligned}
$$

Similarly, if $\mu(y \mid x) \leq \mu(x \mid y)$, then $\mu\left(x y^{*}\right)<\mu(x)$.

Now, if we allow ourselves to permute $x, y, x^{*}, y^{*}$, then we can always arrange that $\mu(y \mid x)$ is minimal among $\left\{\mu(x \mid y), \mu(y \mid x), \mu\left(x \mid y^{*}\right), \mu\left(y \mid x^{*}\right)\right\}$, so that $\max \left\{\mu(x y), \mu\left(x y^{*}\right)\right\}<\mu(x)$.

Lemma 8.1. If $\mu(\mathcal{B} \backslash\{0,1\})$ is well-ordered (as a subset of $S$ ), then $\mathcal{B}$ has a nested set of generators.

Proof. Let $\mathcal{E} \subseteq \mathcal{B} \backslash\{0,1\}$ be the set of $x \in \mathcal{B}$ such that $x$ does not lie in the ring generated by $\{z \in \mathcal{B} \backslash\{0,1\} \mid \mu(z)<\mu(x)\}$. Clearly, $\mathcal{E}$ generates $\mathcal{B}$. Moreover, $\mathcal{E}$ is nested. For if $x, y \in \mathcal{E}$ were not nested, then, without loss of generality, $\max \left\{\mu(x y), \mu\left(x y^{*}\right)\right\}<\mu(x)$. But $x=x y+x y^{*}$, giving a contradiction.

Note that the generating set we obtain is $*$-invariant.

\section{A variation on the Bergman norm.}

In this section, we give a generalisation of Bergman's result [Ber].

Let $V$ be a set, and let $\mathcal{P}(V)$ be its power set. Let $\mathcal{R}=\mathcal{R}(V)$ of binary partitions of $V$, i.e., pairs $\{A, B\} \subseteq \mathcal{P}(V)$ such that $V=A \sqcup B$. We say that $\{A, B\}$ is nontrivial if $A, B \neq \emptyset$. Note that $\mathcal{R}$ has the structure of an abelian group, with addition defined by $\left\{A, A^{*}\right\}+\left\{B, B^{*}\right\}=\{(A \cap B) \cup$ $\left.\left(A^{*} \cap B^{*}\right),\left(A \cap B^{*}\right) \cup\left(A^{*} \cap B\right)\right\}$. The same structure can be obtained by quotienting the additive group of the Boolean $\operatorname{ring} \mathcal{P}(V)$ by the subgroup $\{0,1\}$.

Let $\Psi$ be the set of unordered pairs (i.e., 2-element subsets) of $V$. If $\pi \in \Psi$ and $P=\left\{A, A^{*}\right\}$, we say that $\pi$ crosses $P$ if $\pi \cap A \neq \emptyset$ and $\pi \cap A^{*} \neq \emptyset$. Let $\Psi(P)=\Psi(A)$ be the set of $\pi \in \Psi$ such that $\pi$ crosses $P$.

Suppose that a group $\Gamma$ acts on $V$.

Definition. A (directed) slice of $V$ is a subset $A \subseteq V$, such that $\Phi \cap \Psi(A)$ is finite for every $\Gamma$-orbit, $\Phi$, in $\Pi$.

We refer to $P=\left\{A, A^{*}\right\}$ as an undirected slice. We write $\mathcal{S}(V)$ for the Boolean algebra of directed slices, and $\hat{\mathcal{S}}(V)$ for the additive group of undirected slices. 
Suppose $\left(\Psi_{n}\right)_{n \in \mathbb{N}}$ is a collection of cofinite $\Gamma$-invariant subsets of $\Psi$ with $\Psi=\bigcup \Psi_{n}$. Given $n \in \mathbb{N}$ and $P \in \mathcal{R}$, write $\Psi_{n}(P)=\Psi_{n} \cap \Psi(P)$. Thus, $P \in \mathcal{S}$ if and only if $\Psi_{n}(P)$ if finite for all $P$. We write $K_{n}$ for the graph with vertex set $V$ and edge set $\Psi_{n}$.

Let $\mathbb{N}^{\mathbb{N}}$ be the set of infinite sequences of natural numbers. This has the structure of an ordered abelian group with the lexicographic order. We define a map, $\mu: \hat{\mathcal{S}} \longrightarrow \mathbb{N}^{\mathbb{N}}$ by setting $\mu(P)=\left(\mu_{n}(P)\right)_{n}$, where $\mu_{n}(P)=$ $\left|\Psi_{n}(P)\right|$.

Recall that $V$ is connected if it is the vertex set of some connected $\Gamma$ graph with finite quotient (i.e., some finite union of the $K_{n}$ is connected). We show:

Theorem 9.1. Let $V$ be a connected cofinite $\Gamma$-set. Then $\mu(\hat{\mathcal{S}}) \subseteq \mathbb{N}^{\mathbb{N}}$ is well-ordered, and the map $\mu$ is finite-to-one modulo the action of $\Gamma$.

It will be convenient for the proof to assume that the sets $\Psi_{n}$ are increasing, i.e., $\Psi_{m} \subseteq \Psi_{n}$ whenever $m \leq n$. There is no loss of generality in doing this, for if we set $\Psi_{n}^{\prime}=\bigcup_{m \leq n} \Psi_{m}$, then if $\left(P^{\alpha}\right)_{\alpha \in \mathbb{N}}$ is an infinite sequence of undirected slices which is non-increasing with respect to the order determined by $\left(\Psi_{n}\right)_{n}$, then some subsequence will be non-increasing with respect to the order determined by $\left(\Psi_{n}^{\prime}\right)_{n}$. We may as well also assume that $K_{0}$, and hence every $K_{n}$, is connected. It is easily verified that if $n \in \mathbb{N}$, then $\mu_{n}(P+Q) \leq \mu_{n}(P)+\mu_{n}(Q)$, with equality precisely if no element of $\Psi_{n}$ crosses both $P$ and $Q$.

We now set about the proof of Theorem 9.1.

For the moment, we can forget about the group, $\Gamma$. Let $K$ be a connected graph, with vertex set $V(K)=V$ and edge set $E(K)$. A finite subset, $I \subseteq E(K)$ is separating if $K \backslash I$ is disconnected. (Here, $K \backslash I$ denotes the graph with vertex set $V(K)$ and edge set $E(K) \backslash I$.)

Definition. A cut is a finite nonempty subset, $I \subseteq E(K)$, such that every circuit (or equivalently, cycle) in $K$ contains an even number of edges of $I$.

Thus, every cut is separating. A cut is minimal if it contains no proper subcut. We similarly define minimality for separating sets. It is easily verified that if $I \subseteq E(K)$ is finite, then the following three conditions are equivalent:

(1) $I$ is a minimal cut,

(2) $I$ is a minimal separating set, or

(3) $K \backslash I$ has precisely two connected components.

Note that each cut $I \subseteq K$ determines a nontrivial partition, $P(K, I)=$ $\left\{A, A^{*}\right\}$, where each path from $A$ to $A^{*}$ contains an odd number of edges of $I$. Conversely, each nontrivial partition $P=\left\{A, A^{*}\right\}$ determines the subset, $I=I(K, P)$, of edges which cross from $A$ to $A^{*}$. If $I$ is finite, then it is a 
cut, and we say that $P$ is an (undirected) $K$-break. There is thus a natural bijection between cuts and $K$-breaks.

Given two cuts $I$ and $J$, we write $I+J$ for their symmetric difference. This is also a cut, and the operation agrees with that already defined on the set of partitions. If $J \subseteq I$ is a subcut, then $I \backslash J=I+J$ is also a subcut, and $I=J+(I \backslash J)$. We shall measure the "size" of a cut by its cardinality.

We note:

Lemma 9.2. Suppose $e \in E(K)$ and $n \in \mathbb{N}$. There are finitely many minimal cuts of size $n$ containing $e$.

Proof. Suppose, for contradiction, that the set, $\mathcal{I}$, of such minimal cuts is infinite. Choose $I \subseteq E(K)$ maximal such that $I$ is contained in infinitely many elements of $\mathcal{I}$. Let $\mathcal{J}=\{J \in \mathcal{I} \mid I \subseteq J\}$. Now, $I$ cannot separate $K$ (otherwise $I$ would be a minimal cut, and $\mathcal{J}=\{I\}$ ). Let $\gamma$ be a path in $K \backslash I$ connecting the endpoints of $e$. Now each element of $\mathcal{J}$ contains some edge of $\gamma$, and so some infinite subset of elements of $\mathcal{J}$ all contain the same edge, say $f$, of $\gamma$. But now $I \cup\{f\}$ is contained in infinitely many elements of $\mathcal{J}$ and hence of $\mathcal{I}$, contradicting the maximality of $I$.

Definition. We say that a cut, $I$, is blocklike if every pair of elements of $I$ lie in a minimal subcut of $I$.

Clearly, in Lemma 9.2, one can replace "minimal cut" by "blocklike cut". Every cut can be uniquely decomposed into maximal blocklike cuts. One way to see this is as follows.

Suppose $\Upsilon$ is a finite connected graph. A block of $\Upsilon$ is a maximal 2-vertexconnected subgraph. Two blocks intersect, if at all, in a single vertex. If $e, f \in E(\Upsilon)$ are distinct, then $e, f$ lie in the same block if and only if they lie in some circuit in $\Upsilon$, and if and only if they lie in some minimal separating set. Note that $\Upsilon$ is bipartite (i.e., $E(\Upsilon)$ is a cut) if and only if each of its blocks is bipartite.

Suppose that $K$ is any connected graph, and $I \subseteq E(K)$ is a cut. Let $\Upsilon=\Upsilon(K, I)$ be the graph obtained by collapsing each connected component of $K \backslash I$ to a point. Thus, $\Upsilon$ is a finite connected bipartite graph. There is a canonical surjective map $\phi: K \longrightarrow \Upsilon$. The preimage of every connected subgraph is connected.

Now it is easily checked that the preimage of every cut in $\Upsilon$ is a subcut of $I$. Moreover, every subcut, $J$, arises in this way: $J=\phi^{-1}(\phi J)$. We also see easily that $J$ is minimal if and only if $\phi J$ is minimal (since $K \backslash J$ has the same number of components as $\phi(K \backslash J)=K \backslash \phi J)$. Thus if follows that $J$ is blocklike if and only if $\phi J$ is blocklike. We can thus decompose $I$ canonically by taking the preimages of (the edge sets of) blocks of $\Upsilon$.

If $P$ is a $K$-break, then we shall write $\Upsilon(K, P)=\Upsilon(K, I(K, P))$. The canonical decomposition of $I(K, P)$ gives us a canonical decomposition of $P$ (depending on $K$ ). 
Now suppose that $K^{\prime}$ is another graph on the same vertex set, $V$, with $K \subseteq K^{\prime}$. Let $\phi: K \longrightarrow \Upsilon=\Upsilon(K, P)$ and $\phi^{\prime}: K^{\prime} \longrightarrow \Upsilon^{\prime}=\Upsilon\left(K^{\prime}, P\right)$ be collapsing maps described above. We can obtain $\Upsilon^{\prime}$ from $\Upsilon$ by identifying certain vertices of the same colour and/or adding edges between vertices of different colours. There is thus a natural map, $\psi: \Upsilon \longrightarrow \Upsilon^{\prime}$, such that $\psi \circ \phi=\phi^{\prime} \circ \iota$, where $\iota$ is the inclusion of $K$ in $K^{\prime}$. If we measure the complexity, $c(\Upsilon)$, of a finite graph, $\Upsilon$, by the number of edges in the complementary graph, i.e., $c(\Upsilon)=\frac{1}{2}|V(\Upsilon)|(|V(\Upsilon)|-1)-E(\Upsilon)$, then we see that the map $\psi$ cannot increase complexity. We have $c\left(\Upsilon^{\prime}\right)=c(\Upsilon)$ if and only if $\psi$ is an isomorphism. In this case, the canonical decomposition of $P$ with respect to $K$ is identical to its canonical decomposition with respect to $K^{\prime}$. Note also that in general, $\left|V\left(\Upsilon^{\prime}\right)\right| \leq|V(\Upsilon)| \leq|I(K, P)|+1$.

Suppose now that $V$ is a set with $\left(\Psi_{n}\right)_{n}$ an increasing collection of sets of pairs of $V$ as described above. We thus get an increasing collection of graphs, $\left(K_{n}\right)_{n}$. We suppose that $K_{0}$ (and hence each of these graphs) is connected. If $P$ is any undirected slice, then the sequence of complexities, $c\left(\Upsilon\left(K_{n}, P\right)\right)$, is non-increasing in $n$. Moreover, the cut $I\left(K_{n}, P\right)$ will be minimal for all sufficiently large $n$ (depending on $P$ ). In this case, $\Upsilon\left(K_{n}, P\right)$ consists of a single edge (so its complexity is 0 ).

Suppose that $\mathcal{T} \subseteq \hat{\mathcal{S}}$ is an infinite set of undirected slices with $\mu_{0}(P)=$ $\left|I\left(K_{0}, P\right)\right|$ bounded, by $\nu$, say for $P \in \mathcal{T}$. Thus, for each $P \in \mathcal{T}$ and $n \in \mathbb{N}$, we have $\left|V\left(\Upsilon\left(K_{n}, P\right)\right)\right| \leq\left|V\left(\Upsilon\left(K_{0}, P\right)\right)\right| \leq \mu_{0}(P)+1 \leq \nu+1$, so there are only finitely many possibilities for the graph $\Upsilon\left(K_{n}, P\right)$. Given a graph $\Upsilon$, and $n \in \mathbb{N}$, let $\mathcal{T}(n, \Upsilon)=\left\{P \in \mathcal{T} \mid \Upsilon\left(K_{n}, P\right)=\Upsilon\right\}$. For any fixed $n$, this partitions $\mathcal{T}$ into finitely many subsets. We can therefore choose $\Upsilon=\Upsilon_{0}$ of minimal complexity, $c\left(\Upsilon_{0}\right)$, with the property that for some $n=n_{0}$, say, the set $\mathcal{T}_{0}=\mathcal{T}\left(n_{0}, \Upsilon_{0}\right)$ is infinite. For any $n \geq n_{0}, \Upsilon\left(K_{n}, P\right)=\Upsilon_{0}$ for all but finitely many $P \in \mathcal{T}_{0}$. (Since $c\left(\Upsilon\left(K_{n}, P\right)\right) \leq c\left(\Upsilon\left(K_{n_{0}}, P\right)\right)=c\left(\Upsilon_{0}\right)$, and if we had strict inequality for infinitely many $P$, then we would contradict the minimality of $c\left(\Upsilon_{0}\right)$.)

We now introduce the action of $\Gamma$. Note that by Lemma $9.2, K_{n}$ has only finitely many blocklike cuts of size $s$ modulo $\Gamma$, for any $n, s \in \mathbb{N}$.

Suppose that $\left(P^{\alpha}\right)_{\alpha \in \mathbb{N}}$ is a sequence of undirected slices all lying in distinct $\Gamma$-orbits. Suppose that $\mu\left(P^{\alpha}\right)$ is non-increasing. We want to derive a contradiction.

First note that applying the construction above, with $\mathcal{T}=\left\{P^{\alpha} \mid \alpha \in \mathbb{N}\right\}$, we can assume that, after passing to a subsequence, $\left(P^{\alpha}\right)_{\alpha}$ has the following property. There exist $n_{0} \in \mathbb{N}$ and a finite bipartite graph, $\Upsilon_{0}$, such that if $n_{0} \leq n \leq \alpha$, then $\Upsilon\left(K_{n}, P^{\alpha}\right)=\Upsilon_{0}$. For notational convenience (replacing $\Psi_{0}$ by $\left.\Psi_{n_{0}}\right)$, we can assume that $n_{0}=0$.

Now for each $\alpha$, we decompose the cut $I\left(K_{0}, P^{\alpha}\right)$ into its maximal blocklike subcuts, $I\left(K_{0}, P^{\alpha}\right)=I_{1}^{\alpha}+\cdots+I_{p}^{\alpha}$. Note that $p$ is the number of blocks 
of $\Upsilon_{0}$, and therefore constant. After passing to a subsequence, we can assume that each cut, $I_{i}^{\alpha}$, is the $\Gamma$-image of some fixed cut $J_{i}$, independent of $\alpha$. Let $P_{i}^{\alpha}=P\left(K_{0}, I_{i}^{\alpha}\right)$ and $Q_{i}=P\left(K_{0}, J_{i}\right)$. Thus, $P^{\alpha}=P_{1}^{\alpha}+\cdots+P_{p}^{\alpha}$. Note that since the $P^{\alpha}$ all lie in different $\Gamma$-orbits, we have $p \geq 2$.

Now for each $\alpha, I\left(K_{n}, P^{\alpha}\right)$ is a minimal cut for all sufficiently large $n$. (To see this, note that $K_{0} \backslash I\left(K_{0}, P^{\alpha}\right)$ has finitely many components. Since $\Psi=$ $\bigcup_{n} \Psi_{n}$, we can find $n$ so that any pair of such components are connected by an edge in $K_{n}$. It follows that $K_{n} \backslash I\left(K_{n}, P^{\alpha}\right)$ has precisely two components.) Thus, there is some $n$ (depending on $\alpha$ ) such that the cuts $\left\{I\left(K_{n}, P_{i}^{\alpha}\right)\right\}_{i=1}^{p}$ are not disjoint. Let $n(\alpha)$ be the smallest such $n$, and let $m=\min \{n(\alpha)\}$ $\alpha \in \mathbb{N}\}$. (Thus $m>0$.) If $n<m$, then for all $\beta \in \mathbb{N}$, the cuts $\left\{I\left(K_{n}, P_{i}^{\beta}\right)\right\}_{i=1}^{p}$ are disjoint. Thus, for $n<m$, we have $\mu_{n}\left(P^{\beta}\right)=\mu_{n}\left(P_{1}^{\beta}\right)+\cdots+\mu_{n}\left(P_{p}^{\beta}\right)=$ $\mu_{n}\left(Q_{1}\right)+\cdots+\mu_{n}\left(Q_{p}\right)$, which is independent of $\beta$.

Now for some $\alpha$, the cuts $\left\{I\left(K_{m}, P_{i}^{\alpha}\right)\right\}_{i=1}^{p}$ are no longer disjoint, so this time we get strict inequality: $\mu_{m}\left(P^{\alpha}\right)<\mu_{m}\left(Q_{1}\right)+\cdots+\mu_{m}\left(Q_{p}\right)$. However, for $\beta \geq m$, we have $\Upsilon\left(K_{m}, P^{\beta}\right)=\Upsilon_{0}=\Upsilon\left(K_{0}, P^{\beta}\right)$, and the natural map from $\Upsilon\left(K_{0}, P^{\beta}\right)$ to $\Upsilon\left(K_{m}, P^{\beta}\right)$ is an isomorphism. Thus, the canonical decompositions of $P^{\beta}$ with respect to $K_{0}$ and $K_{m}$ are identical. In particular, the cuts $\left\{I\left(K_{m}, P_{i}^{\beta}\right)\right\}_{i=1}^{p}$ are disjoint, and again, we have equality: $\mu_{m}\left(P^{\beta}\right)=\mu_{m}\left(Q_{1}\right)+\cdots+\mu_{m}\left(Q_{p}\right)$. Thus $\mu_{m}\left(P^{\beta}\right)>\mu_{m}\left(P^{\alpha}\right)$. Taking $\beta>\alpha$, we derive a contradiction to the assumption that $\mu$ is non-increasing.

This proves Theorem 9.1.

We note the following corollary of this result.

Suppose that $V$ is countable. Let $\left(\Psi_{n}\right)_{n}$ be an enumeration of the $\Gamma$-orbits of the set of pairs, $\Psi$. Suppose $A, B \in \mathcal{S}$ are disjoint. Let $\sigma_{n}(A, B)$ be the number of pairs in $\Psi_{n}$ with one element in $A$ and one element in $B$. Let $\sigma(A, B)=\left(\sigma_{n}(A, B)\right)_{n} \in \mathbb{N}^{\mathbb{N}}$. Thus, $\mu(A)=\sigma\left(A, A^{*}\right)$. We are thus in the situation described in Section 8. Applying Lemma 8.1, we deduce:

Theorem 9.3. Suppose $\Gamma$ is a countable group, and $V$ is a connected cofinite $\Gamma$-set. Let $\mathcal{S}$ be the Boolean algebra of slices of $V$. Then, any $\Gamma$-invariant subalgebra of $\mathcal{S}$ has a $\Gamma$-invariant nested set of generators.

Proof. The construction of Section 8 was canonical, and hence $\Gamma$-invariant.

We finish with the following observation with regard to slices.

Lemma 9.4. Suppose that $V$ is a cofinite fine $\Gamma$-set with finite pair stablisers and $W \subseteq V$ is a $\Gamma$-invariant subset. Suppose that each element of $V \backslash W$ has finite stabliser. Then the $\operatorname{map}[A \longrightarrow A \cap W]: \mathcal{S}(V) \longrightarrow \mathcal{S}(W)$ is an epimorphism of $\Gamma$ Boolean algebras. 
Proof. We only really need to note that the map is surjective. To see this, suppose $B \in \mathcal{S}(W)$. Choose any $b \in B$ and any orbit transversal, $\left\{x_{1}, \ldots, x_{n}\right\}$, of $V \backslash W$. Let $A=B \cup\left\{g x_{i} \mid 1 \leq i \leq n, g b \in B\right\}$.

To verify that $A \in \mathcal{S}(V)$, suppose that $x, y \in V$ are distinct. We connect $x$ and $y$ by an edge $e$. Let $E_{0}$ be the set of edges connecting $b$ to each of the $x_{i}$. Let $K$ be the graph with vertex set $V$ and edge set $\Gamma e \cup \cup \Gamma E_{0}$. We see that $K$ is fine.

Now if some $\Gamma$-image, $e^{\prime}$, of $e$ connects $A$ to $A^{*}$, then it lies in an arc of length at most 3 connecting $B$ to $B^{*}$, and whose interior vertices are at $\Gamma$-images of $x$ or $y$. Since $x$ and $y$ have finite degree in $K$, then modulo $\Gamma$, there are only finitely many possibilities for such arcs, and hence for its pair of endpoints. But since $B \in \mathcal{S}(\Gamma)$, there can only be finitely many such arcs in total. Thus, there are only finitely many such edges $e^{\prime}$. Since $x, y$ were arbitrary, we have shown that $A \in \mathcal{S}(V)$.

\section{A finiteness result for Boolean algebras related to simplicial trees.}

In this section, using a result of [DiD], we give a proof of the following:

Lemma 10.1. Suppose that $\Gamma$ is a countable group, and that $T$ is a cofinite simplicial $\Gamma$-tree with finite edge stabilisers. Suppose that $\mathcal{A}$ is any $\Gamma$-invariant Boolean subalgebra of the Boolean algebra, $\mathcal{B}(T)$. Then $\mathcal{A}$ is finitely generated as a $\Gamma$-Boolean algebra.

Here, $\mathcal{B}(T)$ is the Boolean algebra of $T$-breaks, as defined in Section 5 . To say that $\mathcal{A}$ is finitely generated as a $\Gamma$-Boolean algebra means that it has a generating set which is a finite union of $\Gamma$-orbits.

Now, $V=V(T)$ has finite pair stabilisers, and so Theorem 9.3 gives us a $\Gamma$-invariant nested set, $\mathcal{E}$, of generators of $\mathcal{A}$. If we know already that $\mathcal{A}$ is finitely generated, then we can assume that $\mathcal{E}$ is cofinite. It follows that if $e \in E(T)$, the set $\{A \in \mathcal{E} \mid e \in I(A)\}$ is finite. We see that if $x, y \in V$, then $\{A \in \mathcal{E} \mid x \in A, y \notin A\}$ is finite. In particular, if $A, B \in \mathcal{E}$, then $\{C \in \mathcal{E} \mid A \subseteq C \subseteq B\}$ is finite. In other words, $\mathcal{E}$ satisfies the finite interval condition. We can thus identify $\mathcal{E}$ with the directed edge set, $\vec{E}(S)$, of a simplicial tree, $S$. Note that $\Gamma$ acts on $S$ with finite edge stabilisers.

If $x \in V(T)$, then $\{A \in \mathcal{E} \mid x \in A\}$ is a flow on $\mathcal{E}$, and hence determines a flow on $S$. Moreover, from the observation of the previous paragraph, there can be no infinite decreasing sequence in the flow (i.e., any strictly decreasing sequence, $A_{1} \supset A_{2} \supset A_{3} \supset \ldots$ with $A_{i} \in \mathcal{E}$ must terminate). The flow thus arises from a unique vertex of $S$. This therefore defines a $\Gamma$-equivariant map $\phi: V(T) \longrightarrow V(S)$.

Suppose $y \in V(S)$. Let $\mathcal{E}(y) \subseteq \mathcal{E}$ be the set of elements of $\mathcal{E}$ which correspond to directed edges with tail at $y$. Let $\mathcal{E}^{*}(y)=\left\{A^{*} \mid A \in \mathcal{E}(y)\right\}$. If $x \in \bigcap \mathcal{E}^{*}(y) \subseteq V(T)$, then each edge corresponding to an element of 
$\mathcal{E}^{*}(y)$ must point towards $\phi(x) \in V(S)$. It follows that $\phi(x)=y$. From this observation, we see that if $y \notin \phi(V(T))$, then $\cap \mathcal{E}^{*}(y)=\emptyset$, so that $\bigcup \mathcal{E}(y)=V(S)$. If it also happens that $\mathcal{E}(y)$ is finite, then $\bigcup \mathcal{E}(y)$ is the sum (i.e., symmetric difference) of the elements of $\mathcal{E}(y)$. We deduce:

Lemma 10.2. If $y \in V(S) \backslash \phi(V(T))$ has finite degree in $S$, then $\sum_{i=1}^{n} A_{i}=$ 1 in $\mathcal{B}(T)$, where $A_{1} \ldots A_{n}$ are the elements of $\mathcal{E}$ which correspond to those edges with tails at $y$.

This result will be used in the proof of Lemma 10.1.

To prove Lemma 10.1, one can use an accessibility result of Dicks and Dunwoody. Following [DiD], we say that an edge of a $\Gamma$-tree is compressible if its endpoints lie in distinct $\Gamma$-orbits and if its stabiliser is equal to an incident vertex stabiliser. (Such an edge can be "compressed" in the corresponding graph of groups to give a smaller graph.) A $\Gamma$-tree is incompressible if it has no compressible edges.

The following is shown in [DiD] (III 7.5, p. 92):

Proposition 10.3. Let $\Gamma$ be a group. Suppose that $S, T$ are cofinite simplicial $\Gamma$-trees with finite edge stabilisers, and that $S$ is incompressible. If there is a $\Gamma$-equivariant map from $V(T)$ to $V(S)$, then $|V(S) / \Gamma| \leq|V(T) / \Gamma|+$ $|E(T) / \Gamma|$.

(In [DiD] is assumed also that $T$ is incompressible. However, it is clear that one can always collapse $T$ to give another tree, $T^{\prime}$, with this property, and with $V\left(T^{\prime}\right)$ isomorphic as a $\Gamma$-set to a $\Gamma$-invariant subset of $V(T)$. This process can only decrease $|V(T) / \Gamma|$ and $|E(T) / \Gamma|$.)

Alternatively, we can use (the argument of) the "elliptic" case of the accessibility result of Bestvina and Feighn $[$ BesF $]$. This gives a slightly different result:

Proposition 10.4. Suppose that $\Gamma$ is a group and that $S, T$ are cofinite simplicial $\Gamma$-trees without edge inversions. Suppose that $S$ is incompressible, and that every edge stabiliser of $S$ fixes a vertex of $T$. If there is a $\Gamma$ equivariant map from $V(T)$ to $V(S)$, then $|V(S) / \Gamma| \leq \max \{1,5|E(T) / \Gamma|\}$.

In particular, this applies to the case of finite edge-stabilisers. We shall sketch a proof below, which is condensed out of the relevant part of [BesF]. Our direct use of Grushko's Theorem bypasses the use of folding sequences. We begin with some preliminary remarks.

Let $t$ be a graph of groups, and let $\Gamma=\pi_{1}(t)$ be its fundamental group. Thus $\Gamma$ acts on the corresponding Bass-Serre tree, $T$, with quotient the underlying graph $|t|=T / \Gamma$. Given $v \in V(t)$ or $e \in E(t)$, we write $\Gamma(v)=\Gamma_{t}(v)$ or $\Gamma(e)=\Gamma_{t}(e)$ for the corresponding vertex or edge groups. A subgroup of $\Gamma$ is elliptic if it is conjugate into a vertex group. Let $t_{0}$ be the graph of groups with the same underlying graph, and with all vertex and edge groups 
trivial. Thus, $\pi_{1}\left(t_{0}\right)=\pi_{1}(|t|)$ is free of rank $\beta(t)=|E(t)|-|V(t)|+1$. Moreover, there is a natural epimorphism from $\Gamma$ to $\pi_{1}\left(t_{0}\right)$ whose kernel contains $\left\langle\left\langle\bigcup_{v \in V(t)} \Gamma(v)\right\rangle\right\rangle$, where $\langle\langle\rangle$.$\rangle denotes normal closure. In particular, if \Gamma$ is the normal closure of some vertex group, $\Gamma(v)$, then $|t|$ is a tree. We see easily that if every other vertex group is $\Gamma$-conjugate into $\Gamma(v)$, then $\Gamma=\Gamma(v)$. Indeed, if every vertex group is conjugate into a subgroup, $G \leq \Gamma(v)$, then $\Gamma=G=\Gamma(v)$.

Proof of Proposition 10.4. After subdividing the edges of $T$, we obtain a $\Gamma$ tree, $T^{\prime}$, with $V(T) \subseteq V\left(T^{\prime}\right)$, and an equivariant morphism $\phi: T^{\prime} \longrightarrow S$ (which sends each edge of $T^{\prime}$ to an edge or vertex of $S$ ). This descends to a graph-of-groups morphism $\phi: t^{\prime} \longrightarrow s$ (where $\left|t^{\prime}\right|=T^{\prime} / \Gamma$ and $|s|=S / \Gamma$ ) which induces the identity map on $\Gamma=\pi_{1}\left(t^{\prime}\right)=\pi_{1}(s)$. We make a series of observations.

Claim 1. If $v \in V(s) \backslash \phi(V(t))$ and $G \subseteq \Gamma(v)$ is $T$-elliptic, then $G$ is $\Gamma(v)$ conjugate into an incident edge group. This is easily seen by considering the arc connecting $a$ lift of $v$ to $V(S)$ (fixed by $\Gamma(v)$ ) to the $\phi$-image of a fixed point of $G$ in $V(T)$.

In fact, the same argument shows that if $v, w \in V(s) \backslash \phi(V(t))$ are the endpoints of an edge $e \in E(s)$, then any $T$-elliptic subgroup, $G$, of $\langle\Gamma(v) \cup$ $\Gamma(w)\rangle$ is conjugate into an incident edge group adjacent to (but different from) e. In particular, from the $T$-ellipticity hypothesis of the proposition, this applies to $G=\Gamma(e)=\Gamma(v) \cap \Gamma(w)$.

We say that a vertex, $v \in V(s)$ is dead if $v \notin \phi(V(t))$ and if $\Gamma(v)$ is the normal closure of the incident edge groups. Otherwise it is live. We thus decompose $V(s)=V_{D}(s) \sqcup V_{L}(s)$ into dead and live vertices.

Claim 2. $\left|V_{L}(s) \backslash \phi(V(t))\right| \leq \beta(t)-\beta(s)$. To see this, let $\bar{s}$ be the graph of groups with underlying graph $|s|$ obtained by collapsing each edge group in $E(s)$ and each vertex group in $\phi(V(t))$ to the trivial group, and by collapsing each remaining vertex group, $\Gamma_{\bar{s}}(v)$, to the quotient of $\Gamma_{s}(v)$ by the normal closure of its incident edge groups. Thus if $v \in V(\bar{s})$, then $\Gamma_{\bar{s}}(v)$ is nontrivial if and only if $v \in V_{L}(s) \backslash \phi(V(t))$. It follows that $\pi_{1}(\bar{s})$ has at least $\mid V_{L}(s) \backslash$ $\phi(V(t)) \mid+\beta(s)$ nontrivial free factors. Now there is a natural epimorphism from $\pi_{1}\left(t_{0}\right)$ to $\pi_{1}(\bar{s})$. The former group is free of rank $\beta(t)$, and so Claim 2 follows by Grushko's Theorem.

Clearly, $|\phi(V(t))| \leq|V(t)|$ and so $\left|V_{L}(s)\right| \leq|E(t)|-\beta(s)+1$.

Claim 3. Suppose $v \in V_{D}(s)$ and $G$ is an incident edge group. Suppose that every other incident edge group is $\Gamma(v)$-conjugate into $G$. Then $\Gamma(v)=G$. To see this, consider the action of $\Gamma(v)$ on a minimal $\Gamma(v)$-invariant subtree of $T$. Let $r$ be the corresponding graph of groups. Now by the $T$-ellipticity hypothesis, $G$ is conjugate into a vertex group, $\Gamma_{r}(w)$, where $w \in V(r)$. 
Moreover, if $H$ is any other vertex group of $r$, then again $H$ is $T$-elliptic, and hence, by Claim 1, is $\Gamma(v)$-conjugate into an incident edge group to $v$ in $s$. Thus $H$ is $\Gamma(v)$-conjugate into $G$. But now $\Gamma(v)=\langle\langle G\rangle\rangle=\langle\langle\Gamma(w)\rangle\rangle$, and so it follows by the discussion before the proof that $\Gamma(v)=G$. The claim follows.

As an immediate corollary, we see (by the incompressibility of $s$ ) that any such vertex must have degree at least 3 in $s$. In particular, all terminal vertices of $s$ are live.

Claim 4. We cannot have two adjacent dead vertices of degree 2 in $s$. For suppose to the contrary that $e \in E(s)$ has endpoints $v, w \in V_{D}(s)$, both of degree 2. From the remark after Claim 1, we see that, without loss of generality, $\Gamma(e)$ is conjugate into $\Gamma(f)$, where $f \in E(s)$ is the other edge incident on $v$. But now, from Claim 3, we derive the contradiction that $v$ has degree at least 3 .

We now have enough information to bound $|V(s)|$ in terms of the complexity of $|t|$. First note that since every terminal vertex of $s$ is live (Claim 3), the number of such vertices is bounded by Claim 2. Moreover (Claim 2), we have $\beta(s) \leq \beta(t)$. This places a bound on the number of vertices of $s$ of degree at least 3. Finally, Claim 4 together with the bound on live vertices places a bound on the number of vertices of degree 2. More careful bookkeeping shows that if $|s|$ is not a point, then $|V(s)| \leq 5|E(t)|-|V(t)|$. Proposition 10.4 now follows.

Now since, $\beta(s) \leq \beta(t)$, we also get a bound on $|E(s)|=|E(S) / \Gamma|$. In fact, to obtain such a bound, we can weaken the hypotheses slightly:

Corollary 10.5. Let $\Gamma$ be a group and that $S, T$ be cofinite $\Gamma$-trees with finite edge stablisers. Suppose that $\phi: V(T) \longrightarrow V(S)$ is a $\Gamma$-equivariant map, and that each compressible edge of $S$ is incident on some element of $\phi(V(T))$. Then there is a bound on $|E(S) / \Gamma|$ in terms of $|E(T) / \Gamma|$.

Proof. To see this, note that we can obtain a $\Gamma$-tree, $S^{\prime}$, by collapsing down a union of trees, $F$, in $S / \Gamma$, consisting of a union of compressible edges. Moreover, we can assume that if $x \in \phi(V(T))$, then the image of $x$ in $S / \Gamma$ is incident to at most one edge of $F$ that is terminal $S / \Gamma$. (Since collapsing such an edge will be sufficient to render all the other edges incident on $x$ incompressible.) Now, Proposition 10.3 or 10.4 gives a bound on the complexity of $S^{\prime} / \Gamma$. This, in turn, gives a bound on the number of edges of $F$, and hence a bound on the complexity of $S / \Gamma$ as claimed.

We can now set about the proof of Lemma 10.1. Suppose that $\Gamma, T$ and $\mathcal{A}$ are as in the hypotheses. By Theorem 9.3 , there is a $\Gamma$-invariant nested set, $\mathcal{E}$, of generators of $\mathcal{A}$. Now, if $\mathcal{A}$ is not finitely generated as a $\Gamma$-Boolean algebra, then we can find an infinite sequence, $\left(A_{n}\right)_{n \in \mathbb{N}}$, of elements of $\mathcal{E}$ such 
that $A_{n}$ does not lie in the $\Gamma$-Boolean algebra generated by $\left\{A_{i} \mid i<n\right\}$. Let $\Gamma\left(A_{n}\right)$ be the stabiliser of $A_{n}$. After passing to a subsequence, we can assume that $\left|\Gamma\left(A_{n}\right)\right|$ is non-decreasing in $n$. Let $\mathcal{E}_{n}$ be the union of the $\Gamma$ orbits of $\left\{A_{i}, A_{i}^{*} \mid i \leq n\right\}$. Given $A \in \mathcal{E}_{n}$, we write $m(A)=i$ to mean that $A$ or $A^{*}$ lies in the $\Gamma$-orbit of $A_{i}$.

Now fix some $n$. As discussed after the statement of Lemma 10.1, we can identify $\mathcal{E}_{n}$ with the directed edge set of a simplicial tree, $S_{n}$, and there is an equivariant map, $\phi: V(T) \longrightarrow V\left(S_{n}\right)$. Note that $\left|E\left(S_{n}\right) / \Gamma\right|=n$. We claim that $S_{n}$ satisfies the weakened hypotheses of Corollary 10.5. In fact, we show that any vertex whose stabiliser fixes an incident edge must lie in $\phi(V(T))$.

Suppose, to the contrary, that $y \in V\left(S_{n}\right) \backslash \phi(V(T))$ is incident on an edge $\vec{e} \in \vec{E}\left(S_{n}\right)$ with tail at $y$ and with $\Gamma(e)=\Gamma(y)$. Note that $\Gamma(y)$ is finite, so that $y$ has finite degree. Let $A \in \mathcal{E}_{n}$ be the element corresponding to $\vec{e}$, so that $\Gamma(A)=\Gamma(y)$. Let $\mathcal{E}_{n}(y)$ be the set of elements of $\mathcal{E}_{n}$ which correspond to edges with tails at $y$. We can assume that $\vec{e}$ is chosen so that $m(A)$ is maximal among those elements of $\mathcal{E}_{n}(y)$ with stabilisers equal to $\Gamma(y)$. Write $\mathcal{E}_{n}(y)=\left\{A, B_{1}, \ldots, B_{k}\right\}$. By Lemma 10.2 , we have that $A=1+\sum_{j=1}^{k} B_{j}$ in the Boolean algebra $\mathcal{B}(T)$. In particular, $A$ lies in the Boolean algebra generated by $\left\{B_{1}, \ldots, B_{k}\right\}$.

Now, for each $j, \Gamma\left(B_{j}\right) \leq \Gamma(A)$. Either $\Gamma\left(B_{j}\right)=\Gamma(A)$ so that, by the maximality of $m(A)$, we have $m\left(B_{j}\right)<m(A)$, or else $\left|\Gamma\left(B_{j}\right)\right|<|\Gamma(A)|$ so that, by the construction of the sequence $\left(A_{i}\right)_{i}$, we again have $m\left(B_{j}\right)<$ $m(A)$. We therefore deduce that $A$ lies in the $\Gamma$-Boolean algebra generated by $\left\{A_{i} \mid i<m(A)\right\}$, contrary to the construction of $\left(A_{i}\right)_{i}$. This proves the claim.

Now applying Corollary 10.5, we get a bound on the complexity $n=$ $\left|E\left(S_{n}\right) / \Gamma\right|$. But we could have chosen $n$ arbitrarily large, thereby giving a contradiction.

This proves Lemma 10.1.

\section{An application to 1-connected $\Gamma$-sets.}

In this section, we apply Lemma 10.1 to show:

Lemma 11.1. Suppose $\Sigma$ is a countable $\mathbb{Z}_{2}$-acyclic simplicial 2-complex which is locally finite away from the vertex set $V(\Sigma)$. Suppose a group $\Gamma$ acts on $\Sigma$ with finite quotient and such that $\Gamma(x) \cap \Gamma(y)$ is finite for all distinct $x, y \in V(\Sigma)$. Let $\mathcal{S}(V(\Sigma))$ be the Boolean algebra of slices of $V(\Sigma)$. Then, any $\Gamma$-invariant subalgebra, $\mathcal{A}$, of $\mathcal{S}(V)$ has a cofinite nested set of generators which is discrete as a protree.

We can reduce Lemma 11.1 to Lemma 10.1 using the machinery of patterns and tracks as in [Du2]. (The overall strategy of the proof is thus 
similar to that of the accessibility result of $[\mathbf{B e s F}]$.) Let $\Sigma, \Gamma, V, \mathcal{A}$ be as in the hypotheses, and let $K$ be the 1-skeleton of $\Sigma$. Recall that a pattern, $t$, on $\Sigma$ is a compact subset of $\Sigma \backslash V(\Sigma)$ which meets each 1-simplex either in the empty set or a single point, and which meets each 2 -simplex, $\sigma$, either in the empty set or in a single arc connecting two distinct faces of $\sigma$. It represents a subset, $A \subseteq V(\Sigma)$ if it meets precisely those edges of $\Sigma$ which connect $A$ to $A^{*}$. Every $\Sigma$-slice is represented by a pattern. A track is a connected pattern. Two disjoint tracks, $s, t$, are parallel if there is a closed subset of $\Sigma \backslash V(\Sigma)$ homeomorphic to $s \times[0,1] \cong t \times[0,1]$ with boundary $s \sqcup t$. If $\mathcal{T}$ is a $\Gamma$-equivariant set of disjoint pairwise non-parallel tracks, then there is a bound on $|\mathcal{T} / \Gamma|$, (see [Du2]).

By Theorem 9.3 there is a $\Gamma$-invariant nested set of generators, $\mathcal{E}$, for $\mathcal{A}$. By a standard construction (cf. [Du2]), we can find a set of patterns $(t(A))_{A \in \mathcal{E}}$ such that $t(A)$ represents $A, t(A)=t\left(A^{*}\right), t(A) \cap t(B)=\emptyset$ if $B \neq A, A^{*}$ and $t(g A)=g t(A)$ for all $g \in \Gamma$. By the observation of the previous paragraph, we can find a cofinite $\Gamma$-invariant set, $\mathcal{T}$, of tracks such that if $A \in \mathcal{E}$, then each connected component of the pattern $t(A)$ is parallel to some element of $\mathcal{T}$. Now $\mathcal{T}$ determines a simplicial tree, $T$, whose edges are in bijective correspondence with $\mathcal{T}$, and whose vertices are in bijective correspondence with the connected components of $\Sigma \backslash \bigcup \mathcal{T}$. (It is here that we use the fact that $\Sigma$ is $\mathbb{Z}_{2}$-acyclic, so that every track separates $\Sigma$.) There is a canonical map $\phi: V(\Sigma) \longrightarrow V(T)$, where two vertices of $\Sigma$ get mapped to the same vertex of $T$ if and only if they are not separated by any element of $\mathcal{A}$. Note that $\Gamma$ acts with finite edge stabilisers on $T$.

Suppose that $A \in \mathcal{E}$. Let $I_{A} \subseteq E(T)$ be the set of edges of $T$ that correspond to the connected components of $t(A)$. Now $I_{A}$ in turn determines an element, $B(A) \in \mathcal{B}(T)$, with the property that $I(B(A))=I_{A}$ and such that $\phi(A) \subseteq B(A)$. It follows that $A=\phi^{-1}(B(A))$. Let $\mathcal{F}=\{B(A) \mid A \in$ $\mathcal{E}$ \}. Thus, $\mathcal{F}$ is a nested subset of $\mathcal{B}(T)$. By Lemma 10.1 , some cofinite $\Gamma$ invariant subset of $\mathcal{F}$ is sufficient to generate the Boolean algebra generated by $\mathcal{F}$. The corresponding elements of $\mathcal{E}$ now generate $\mathcal{A}$. (This follows because $A=\phi^{-1}(B(A))$ for all $A \in \mathcal{E}$, so that any relation between the elements of $\mathcal{F}$ also holds between the corresponding elements of $\mathcal{E}$.) We see that $\mathcal{A}$ has a cofinite generating set, as required. This proves Lemma 11.1.

Corollary 11.2. Let $V$ be a fine $\mathbb{Z}_{2}$-homologically 1 -connected $\Gamma$-set with finite pair stabilisers. Then any $\Gamma$-invariant subalgebra of $\mathcal{S}(V)$ has a cofinite nested set of generators which is discrete as a protree.

Proof. As described in Section $2, V$ can be embedded in a $\mathbb{Z}_{2}$-acyclic 2complex which is locally finite away from $V$. Let $\mathcal{A}^{\prime}=\{A \in \mathcal{S}(V(\Sigma)) \mid$ $V \cap A \in \mathcal{A}\}$. By Lemma 9.4, the map $[A \longrightarrow V \cap A]$ is an epimorphism from $\mathcal{A}^{\prime}$ to $\mathcal{A}$. A nested set of generators for $\mathcal{A}^{\prime}$ as given by Lemma 11.1 then gives us the required generating set for $V$. 


\section{Convergence actions on Cantor sets in the finitely presented case.}

In this section, we shall give proofs of the main results stated in Section 1.

Suppose the group $\Gamma$ acts as a convergence group on the Cantor set, $M$. We write $\Pi \subseteq M$ for the set of non-conical limit points, and $\mathcal{B}(M)$ for the Boolean algebra of clopen sets of $M$. We suppose that there is a cofinite $\Gamma$-invariant collection, $\mathcal{G}$, of parabolic subgroups of $\Gamma$ such that $\Gamma$ is almost finitely presented relative to $\mathcal{G}$. As a $\Gamma$-set, $\mathcal{G}$ is isomorphic to a $\Gamma$-invariant subset, $\Pi_{0}$, of $\Pi$. By Lemma $4.2, \Pi_{0}$ is fine.

Firstly consider the case where $\Pi_{0} \neq \emptyset$. By hypothesis, $\Pi_{0}$ is $\mathbb{Z}_{2}$-homologically 1-connected. Let $\mathcal{A}=\left\{A \cap \Pi_{0} \mid A \in \mathcal{B}(M)\right\}$. Since $\Pi_{0}$ is dense in $M, \mathcal{A}$ is isomorphic to $\mathcal{B}(M)$ as a $\Gamma$-Boolean algebra, and so $\Xi(\mathcal{A})$ is $\Gamma$-equivariantly homeomorphic to $\Xi(\mathcal{B}(M)) \cong M$. Moreover, applying Lemma 4.1, we see that $\mathcal{A}$ is a subalgebra of $\mathcal{S}\left(\Pi_{0}\right)$. By Corollary 11.2, $\mathcal{A}$ has a cofinite $\Gamma$-invariant nested set of generators, $\mathcal{E}$, which is discrete as a protree. It can thus be identified with the directed edge set of a cofinite simplicial tree, $T$, with finite edge stabilisers. We can construct the space $\Xi(\mathcal{E})$ as in Section 7 , where $\mathcal{E}$ is viewed as a protree. As in Section 6 , we see that $\Xi(\mathcal{E})$ be indentified with $\Delta T$, and so $\Delta_{0} T$ is precisely $\Xi(\mathcal{E})$ with its isolated points removed. Moreover, in Section 7 , we saw that $\Xi(\mathcal{A})$ can be canonically embedded in $\Xi(\mathcal{E})$ so that every point of $\Xi(\mathcal{E}) \backslash \Xi(\mathcal{A})$ is isolated. Since $\Xi(\mathcal{A}) \equiv M$ is a Cantor set, we see that $M$ can also be identified with $\Xi(\mathcal{E})$ with its isolated points removed, and hence with $\Delta_{0} T$.

Now, $\Gamma$ is hyperbolic relative to the infinite vertex stabilisers and its boundary as a relatively hyperbolic group is precisely $\Delta_{0} T$ (see Section 6). We have thus shown that the boundary is $\Gamma$-equivariantly homeomorphic to $M$. (In retrospect, we see that $\Pi$ is precisely the set of parabolic points.)

In the case where $\Pi_{0}=\emptyset$, we can find a $\mathbb{Z}_{2}$-acyclic complex on which $\Gamma$ acts freely. If $\Pi \neq \emptyset$, we take a $\Gamma$-equivariant map $\phi: V(\Sigma) \longrightarrow \Pi$. Again, the image of $\phi$ is a fine $\Gamma$-set. Let $\mathcal{A}$ be the Boolean algebra $\left\{\phi^{-1} A \mid A \in \mathcal{B}(M)\right\}$. Again, $\mathcal{A}$ is a subalgebra of $\mathcal{S}(V)$ isomorphic to $\mathcal{B}(M)$ and the argument proceeds as before.

It remains to deal with the case where $\Pi=\emptyset$, in other words, every point of $M$ is a conical limit point. By [Bo1] it follows that $\Gamma$ is hyperbolic with boundary $M$. It is now an easy consequence of Dunwoody's accessibility theorem $[\mathbf{D u} 2]$ that $\Gamma$ is virtually free and that $M \equiv \partial \Gamma$ may be identified with the space of ends of $\Gamma$.

This concludes the proofs of Theorems 1.4 and 1.1. We immediately deduce Theorem 1.3. Theorem 1.2 follows from Theorem 1.4 and the discussion at the end of Section 5 . 


\section{Convergence actions arising from protrees.}

Suppose $\Theta$ is a $\Gamma$-protree such that the stabiliser of each element is finite. We say that $\Theta$ is locally discrete if each cofinite subprotree is discrete. Note that $\Gamma$ acts on $\Xi(\Theta)$ by homeomorphism.

Proposition 13.1. If $\Theta$ is locally discrete, then $\Gamma$ acts on $\Xi(\Theta)$ as a convergence group.

Proof. By definition, $\Xi(\Theta)$ is dual to the Boolean ring $\mathcal{F}(\Theta)$ defined in Section 7. It is easily verified that the action of $\Gamma$ on $\mathcal{F}(\Theta)$ is a convergence action, as defined at the end of Section 4.

If $\Theta$ is countable, then we can write it as an increasing union, $\Theta=$ $\bigcup_{n=1}^{\infty} \Theta_{n}$, of cofinite discrete $\Gamma$-protrees, $\Theta_{n}$. We can identify $\Theta_{n}$ as the directed edge set of a simplicial $\Gamma$-tree, $T_{n}$. We see that $\Xi(\Theta)$ is an equivariant inverse limit of the spaces $\Xi\left(\Theta_{n}\right) \cong \Delta T_{n}$, and that $\Xi_{0}(\Theta)$ is an equivariant inverse limit of the spaces $\Delta_{0} T_{n}$. (This gives another proof of the fact that $\Gamma$ acts as a convergence group on $\Xi(\Theta)$.) We see that the action of $\Gamma$ on $\Xi(\Theta)$ is an inverse limit of geometrically finite actions.

Note that an inaccessible group admits a locally discrete action on a nondiscrete protree. Dunwoody's example of a finitely generated inaccessible group [Du3] thus gives an example of a non-geometrically finite action of such a group on a Cantor set.

We show that examples of this type are typical of convergence actions of (relatively) finitely generated groups on Cantor sets:

Theorem 13.2. Suppose that $\Gamma$ acts as a minimal convergence group on a Cantor set, $M$, and that $\mathcal{G}$ is a finite collection of parabolic subgroups. Suppose that $\Gamma$ is finitely generated relative to $\mathcal{G}$. Then, $\Gamma$ admits a locally discrete action on a countable protree, $\Theta$, such that $M$ is equivariantly homeomorphic to $\Xi(\Theta)$.

In particular, the action of $\Gamma$ on $M$ is an inverse limit of geometrically finite actions.

The proof of Theorem 13.2 proceeds exactly as with that of Theorem 1.4 (and 1.1) as described in Section 12, except that, in this case, we have to make do with a (fine) connected cofinite $\Gamma$-graph, $K$, instead of the 2complex, $\Sigma$. As before, $M$ is equivariantly homeomorphic to $\Xi(\mathcal{A})$ where $\mathcal{A}$ is a $\Gamma$-subalgebra of the Boolean algebra of $K$-breaks. Theorem 9.3 gives us an invariant nested generating set, $\mathcal{E}$ of $\mathcal{A}$, which has the structure of a protree, $\Theta$, as described in Section 7. We can canonically identify $\Xi(\Theta)$ as a closed subset of $\Xi(\mathcal{A}) \cong M$, whose complement consists of isolated points and is thus empty in this case. We have equivariantly identified $\Xi(\Theta)$ with $M$ as required. 


\section{Other applications.}

In this section, we sketch two further applications of Theorem 9.3. One concerns constructions of group splittings, and the other relates to the Almost Stability Theorem of [DiD].

Suppose that $\Gamma$ is a one-ended finitely generated group, and that $G \leq \Gamma$ is any subgroup. Let $X$ be a Cayley graph of $\Gamma$ (or any cofinite locally finite $\Gamma$-graph). As in [Bo4] (cf. $[\mathbf{D u S}]$ ) we say that $G$ has codimension-one in $\Gamma$ if there is a connected $G$-invariant subset, $Y \subseteq X$, such that $Y / G$ is compact, and such that $X \backslash Y$ has at least two distinct components neither of which is contained in a uniform neighbourhood of $Y$. (This is independent of the choice of $X$.) The following result also follows directly from a result of Niblo $[\mathbf{N}]$ :

Proposition 14.1. Suppose that $\Gamma$ is finitely generated and that $G \leq \Gamma$ is a codimension-one subgroup such that $\Gamma$ is the commensurator of $G$. Then, $\Gamma$ splits nontrivially as a graph of groups with $G$ conjugate into one of the vertex groups.

The "commensurator" condition means that $G \cap g G g^{-1}$ has finite index in $G$ for all $g \in \Gamma$. If we assume that no vertex group is equal to an incident edge group, then it follows that all the vertex and edge groups will be commensurate with $G$.

To prove Proposition 14.1, let $K$ be a coset graph of $G$ in $\Gamma$. In other words, $K$ is a connected cofinite $\Gamma$-graph, with $V(K)$ isomorphic as a $\Gamma$-set to the set of left translates of $G$, with $\Gamma$ acting by left multiplication. Let $x \in V(K)$ be a vertex stabilised by $G$. Let $Y \subseteq X$ be as in the hypotheses. Thus, we can write $X \backslash Y=P \sqcup Q$ where neither $P$ nor $Q$ is contained in any uniform neighbourhood of $Y$. Now all but finitely many $\Gamma$-images of $Y$ are disjoint from $Y$, and hence contained in either $P$ or $Q$. Each $\Gamma$-image of $Y$ corresponds to a vertex of $K$. This therefore assigns all but finitely many elements of $V(K)$ to one of two disjoint infinite subsets, $A, B \subseteq V(K)$, corresponding to $P$ and $Q$ respectively. Assigning the remaining vertices arbitrarily, we can suppose that $B=A^{*}$.

If $y \in A$ and $z \in A^{*}$, then any path connecting the corresponding images of $Y$ in $X$ must intersect $Y$. Since $Y / G$ is finite, it follows that only finitely many $\Gamma$-images of any pair $\{y, z\} \subseteq V(K)$ can meet both $A$ and $A^{*}$. In other words, $A$ is a slice. We have shown that the algebra of slices, $\mathcal{B}(K)$, of $K$ is nontrivial, Theorem 9.3 now applies to give us a nested set of generators for $\mathcal{B}(K)$. Since the set of $\Gamma$-images of $Y$ is locally finite in $X$, it follows that this generating set is locally discrete. We thus get an action of $\Gamma$ on a simplicial tree, $T$. Moreover, there is an equivariant map from $V(K)$ to $V(T)$. This proves Proposition 14.1. 
This result is, in some sense, "orthogonal" to the constructions of [Bo5]. Note that we cannot expect the splittings we obtain in this case to be canonical.

Another application of Theorem 9.3 (as observed by Dunwoody) is to give an alternative proof of a version of the Almost Stability Theorem of [DiD]. This can be interpreted as giving a criterion for a $\Gamma$-set to be enbedded in the vertex set of a simplicial $\Gamma$-tree with finite edge stabilisers. Let $\Gamma$ be a group, and $X$ be a cofinite $\Gamma$-set. Let $\mathcal{P}(X)$ be the power set of $X$, thought of as a $\Gamma$-Boolean algebra, and let $\mathcal{I}$ be the ideal of finite subsets.

Proposition 14.2. Suppose that $\Gamma$ is a finitely generated group, and that $X$ is a $\Gamma$-set with finite point stabilisers. Suppose that $V \subseteq \mathcal{P}(X)$ is a $\Gamma$ invariant subset of $\mathcal{P}(X)$ with the property that if $A, B \in V$ then $A+B \in \mathcal{I}$. Then $V$ can be equivariantly embedded in the vertex set of a simplicial $\Gamma$-tree.

Suppose we already know that $V$ embeds in a simplicial $\Gamma$-tree, $T$. We can take $X$ to be the directed edge set of $T$. To each $x$ in $V$, we associate the set of directed edges which point towards $x$. This gives a subset of $\mathcal{P}(X)$ satisfying the hypotheses of Proposition 13.2. Of course, the situation described by the hypotheses may be more general that this.

Proposition 14.2 can be proven as follows. Given any $x \in X$, let $A(x) \in$ $\mathcal{P}(V)$ be the subset of $V$ consisting of those elements of $V$ that contain $x$. One verifies that $A(x)$ is a slice of $V$. Let $\mathcal{A}$ be the subalgebra of slices of $V$ generated by $\{A(x) \mid x \in V\}$. (A typical element of $\mathcal{A}$ has the form $B$ or $B^{*}$, where $B$ is either finite or is equal to $A(x)$ for some $x \in X$.) We now apply Theorem 9.3. It is easily verified that the resulting generating set is locally discrete.

\section{References}

[Ber] G.M. Bergman, On groups acting on locally finite graphs, Ann. of Math., $8 \mathbf{8}$ (1968), 335-340, MR 37 \#4154, Zbl 0164.02801.

[BesF] M. Bestvina and M. Feighn, Bounding the complexity of simplicial group actions on trees, Invent. Math., 103 (1991), 449-469, MR 92c:20044, Zbl 0724.20019.

[Bo1] B.H. Bowditch, A topological characterisation of hyperbolic groups, J. Amer. Math. Soc., 11 (1998), 643-667, MR 99c:20048, Zbl 0906.20022.

[Bo2] - Connectedness properties of limit sets, Trans. Amer. Math. Soc., 351 (1999), 3673-3686, MR 2000d:20056, Zbl 0938.20033.

[Bo3] Convergence groups and configuration spaces, in 'Group Theory Down Under' (ed. J. Cossey, C.F. Miller, W.D. Neumann and M. Shapiro), de Gruyter, 1999, 23-54, MR 2001d:20035, Zbl 0952.20032. 
[Bo4] Splittings of finitely generated groups over two-ended subgroups, Trans. Amer. Math. Soc., 354 (2002), 1049-1078, CMP 1867372.

[Bo5] _ Relatively hyperbolic groups. Preprint, Southampton, 1997.

[CJ] A. Casson and D. Jungreis, Convergence groups and Seifert fibered 3-manifolds, Invent. Math., 118 (1994), 441-456, MR 96f:57011, Zbl 0840.57005.

[DiD] W. Dicks and M.J. Dunwoody, Groups Acting on Graphs, Cambridge Studies in Advanced Mathematics, 17, Cambridge University Press, 1989, MR 91b:20001, Zbl 0665.20001.

[Du1] M.J. Dunwoody, Cutting up graphs, Combinatorica, 2 (1982), 15-23, MR 84k:05050, Zbl 0504.05035.

[Du2] , The accessibility of finitely presented groups, Invent. Math., 81 (1985), 449-457, MR 87d:20037, Zbl 0572.20025.

[Du3] _ An inaccessible group, in 'Geometric Group Theory,' Vol. 1 (ed. G.A. Niblo and M.A. Roller), London Math. Soc. Lecture Notes Series, 181, Cambridge University Press, 1993, 75-78, MR 94i:20067, Zbl 0833.20035.

[Du4] Groups acting on protrees, J. London Math. Soc., 56 (1997), 125-136, MR 98f:20008, Zbl 0918.20011.

[DuS] M.J. Dunwoody and E.L. Swenson, The algebraic torus theorem, Invent. Math., 140 (2000), 605-637, MR 2001d:20039.

[F] E.M. Freden, Negatively curved groups have the convergence property, Ann. Acad. Sci. Fenn. Ser. A Math., 20 (1995), 333-348, MR 96g:20054, Zbl 0847.20031.

[Ga] D. Gabai, Convergence groups are fuchsian groups, Ann. of Math., 136 (1992), 447-510, MR 93m:20065, Zbl 0785.57004.

[GehM] F.W. Gehring and G.J. Martin, Discrete quasiconformal groups I, Proc. London Math. Soc., 55 (1987), 331-358, MR 88m:30057, Zbl 0628.30027.

[Ger] V. Gerasimov, Manuscript (Russian), Belo Horizonte.

[Gr] M. Gromov, Hyperbolic groups, in 'Essays in Group Theory' (ed. S.M. Gersten), M.S.R.I. Publications, 8, Springer-Verlag, 1987, 75-263, MR 89e:20070, Zbl 0634.20015.

[N] G.A. Niblo, The singularity obstruction for group splittings, Topology Appl., 119(1) (2002), 17-31, CMP 1881707.

[Si] G.F. Simmons, Introduction to Topology and Modern Analysis, McGraw-Hill, 1963, MR 84b:54002, Zbl 0105.30603.

[St] M.H. Stone, Applications of the theory of Boolean rings to general topology, Trans. Amer. Math. Soc., 41 (1937), 375-481, CMP 1501 905, Zbl 0017.13502.

[T1] P. Tukia, Homeomorphic conjugates of fuchsian groups, J. Reine Angew. Math., 391 (1988), 1-54, MR 89m:30047, Zbl 0644.30027.

[T2] Convergence groups and Gromov's metric hyperbolic spaces, New Zealand J. Math., 23 (1994), 157-187, MR 96c:30042, Zbl 0855.30036.

[T3] Conical limit points and uniform convergence groups, J. Reine. Angew. Math., 501 (1998), 71-98, MR 2000b:30067, Zbl 0909.30034.

[Y] A.Yaman, A topological characterisation of relatively hyperbolic groups. Preprint, Southampton, 2001.

Received February 10, 2001 and revised September 20, 2001. 
FaCUlty of Mathematical Studies

UNIVERSITY OF SOUTHAMPTON

Highfield, Southampton SO17 1BJ

Great Britain

E-mail address: B.H.Bowditch@maths.soton.ac.uk 